\title{
Inverse identification strategies for the characterization of transformation-based anisotropic plasticity models with the non-linear VFM
}

\author{
Attilio Lattanzi ${ }^{\mathrm{a}, *}$, Frédéric Barlat ${ }^{\mathrm{b}}$, Fabrice Pierron ${ }^{\mathrm{c}}$, Aleksander Marek $^{\mathrm{c}}$, \\ Marco Rossia \\ ${ }^{a}$ Department of Industrial Engineering and Mathematics, Università Politecnica delle \\ Marche, via Brecce Bianche, Ancona, 60131, Italy \\ ${ }^{b}$ Graduate Institute of Ferrous Technology (GIFT), Pohang University of Science and \\ Technology (POSTECH), 7 ry Cheongam-ro, Nam-gu, Pohang, Gyeongbuk 37673, \\ Republic of Korea \\ ${ }^{c}$ Faculty of Engineering and Physical Sciences, University of Southampton, Highfield, \\ SO171BJ, UK
}

\begin{abstract}
The Virtual Fields Method (VFM) is a well established inverse technique used to identify the constitutive parameters of material models using heterogeneous full-field strain data. When VFM is employed to retrieve the coefficients of advanced plasticity models, including non linear hardening and anisotropy, however, the procedure may become computationally intensive. Furthermore, the impact of experimental uncertainties is still not entirely scrutinized. In this paper, an identification strategy based on uncoupling the hardening behaviour and the anisotropic yield function is proposed. The approach, based on VFM, allows to carry on the identification with low com-

\footnotetext{
${ }^{*}$ Corresponding author

Email addresses: a.lattanzi@staff.univpm.it (Attilio Lattanzi), f.barlat@postech.ac.kr (Frédéric Barlat), f.pierron@soton.ac.uk (Fabrice Pierron), a.marek@soton.ac.uk (Aleksander Marek), m.rossi@staff .univpm.it (Marco Rossi)
} 
putational time, and provides also indications on the optimal smoothing level to use in the full-field measurement.

The identification framework is applied on the linear transformationbased yield condition Yld2000-2D, employing numerical data for the validation and, afterwards, using actual experimental data on a bake-hardenable steel, i.e. BH340. Moreover, several aspects of the identification procedure are investigated in dept, namely, the effect of smoothing, the influence of VFM settings (type of virtual fields used, discretization method) and the computational time. The identification results are compared with the standard calibration process, demonstrating that the proposed strategy is capable of identifying properly the material anisotropic behaviour using only three tests on notched specimens.

Keywords: Anisotropic plasticity, Inverse Identification, Virtual Fields Method, Digital Image Correlation, Large deformations

\section{Introduction}

The application of the Finite Element (FE) modelling to simulate manufacturing like sheet metal forming, represents a powerful tool to design processes and products with enhanced structural performances and quality standards, which are conform to the modern automotive industry requirements. However, the accuracy given by FE predictions depends on a number of simulation parameters and is also intrinsically related to the constitutive model adopted to describe the material response. In particular, the sheet metals anisotropy is a key feature that can control the success of the simulation results. In fact, the rolling process induces a thermo-mechanical 
deformation on the sheet metal, which leads to preferential orientations in the material texture [1, 2] often expressible as an orthotropic symmetry.

This microstructural character obviously reflects on the material behaviour at macroscopic level, that is captured and described by an appropriate yield condition. Several plastic anisotropy models were developed according to different approaches, based, for example, on the definition of common stress invariants and structural tensors of plastic anisotropy, or on the introduction of transformations of the stress tensor [3]. In particular, this latter method is the foundation of many constitutive models, where the anisotropic behaviour is described by means of linear transformation tensors [4 8]. Among them, the Yld2000-2D and the Yld2004-18p yield functions gained particular interest for industrial applications because of their robustness and ability to reproduce the material anisotropic response, as for the prediction of cupearing profile [9-11] and forming limit diagrams [12].

Nonetheless, a large number of experimental tests is required to calibrate their constitutive parameters, including data from the uniaxial and biaxial tests. The equibiaxial tension can be achieved by performing multiaxial test on cruciform specimens [13] or via hydraulic bulge test [14], demanding specific testing equipment that are not always available in industrial labs, consequently increasing the costs for a complete characterization.

Recently, the rapid diffusion of full-field measurement techniques like the Digital Image Correlation (DIC) [15] was followed by the development of methods to inversely calibrate even complex material models [16, 17]. The use of heterogeneous strain fields, in fact, allows the gathering of a larger amount of information about the material state from a single test, permit- 
ting the reduction of the experimental efforts to characterize the constitutive model [18]. The Finite Element Model Updating (FEMU), for instance, represents a widely diffused method, where the test is simulated by varying iteratively the material parameters until the difference between experimentally measured variables (i.e. loading force, strain fields) and the numerical results is minimized. In plasticity FEMU was already applied to determine the hardening behaviour of Domex 650 [19], and to calibrate anisotropic yielding functions such as Hill48 [20, 21] and Yld2000-2D [22] models. In [23], the weighted FEMU is compared with another inverse method, the Integrated Digital Image Correlation (I-DIC), to identify the Ramberg-Osgoord model. Based on the global DIC, whose nodal displacement and constitutive parameters are considered unknown and mutually determined by the identification procedure, the I-DIC was also applied to characterize the Hill48 model in 24] using biaxial test on cruciform specimen.

There are alternative identification approaches that do not employ FE simulations, increasing the computational efficiency of the whole process [25, 26]. The Virtual Fields Method (VFM) [27], belongs to this class of inverse techniques, and it has been successfully implemented in many non-linear plasticity problems in the case of isotropic [28] and anisotropic behaviour [2932]; Pierron et al. [33] applied the VFM to cyclic plasticity with kinematic hardening, and $\mathrm{Fu}$ et al. 34] to the $\mathrm{HAH}$ distortional plasticity model.

The accuracy of the identification results achieved with VFM is influenced by the adopted virtual fields (VFs) because of their interaction with experimental uncertainties. The VFs can be arbitrarly chosen by the user or selected using dedicated procedures. For instance, Avril et al. 35] proposed 
a procedure to generate automatically the VFs minimizing the sensitivity to noise. Recently, Marek et al. in [36] developed a VFs generation method based on the sensitivity of the reconstructed stress field to the constitutive parameters. Although such method outperformed the traditional manual VFs for calibrating the Yld2000-2D coefficients [36, only numerical data with synthetic noise were adopted in that study, and the whole process to identify both hardening law and yielding function were computationally intensive.

The present study proposes a novel inverse identification protocol for large deformations problems based on the VFM, and is addressed to provide a robust and reliable strategy capable of identifying correctly the sought constitutive parameters. In particular, the main purposes of this paper are:

- simplify the experimental set-up and reducing the experimental effort required by the standard calibration method;

- increase the computational efficiency of the whole inverse identification process;

- provide effective criteria to define the VFM settings and to manipulate the input data.

Thereby, the proposed procedure basically decouples the identification of the hardening behaviour from the anisotropic plasticity model: the former is achieved by using the linear stress-strain curve identification (LSSCI) 37], while the latter relies on the non-linear VFM technique. This approach is possible for the case where isotropic hardening is assumed

The identification strategy is exploited to identify the coefficients of Yld2000$2 \mathrm{D}$ on computer-generated test data and, then, on real experiments per- 
formed on bake-hardenable $(\mathrm{BH})$ steel for automotive applications, namely, BH340.

\section{Theoretical Framework}

\subsection{The non-linear VFM for large deformations problems}

Continuum mechanics problems are founded on the equilibrium equations and the relationship between displacements, strains and stresses, which must be defined for all material points based on the kinematics. Let us introduce, in the Euclidean space, a body $\mathcal{B}$ subjected to a general deformation process at time $t$. According to the finite deformation theory [38], it is possible to distinguish between its reference placement $\mathcal{B}_{0}$ and its current placement $\mathcal{B}_{t}$, as illustrated in Figure 1. Considering a single material point $P$, its position in the reference configuration is indicated by the vector $\mathbf{x}_{0}$, while its placement in the current configuration is represented by the position vector $\mathbf{x}$. The bijective function $\chi$ is introduced in order to describe the motion of $P$, so that $\mathbf{x}=\boldsymbol{\chi}\left(\mathbf{x}_{0}, t\right)$; thereby, the displacement vector is defined as:

$$
\mathbf{u}\left(\mathbf{x}_{0}, t\right)=\mathbf{x}-\mathbf{x}_{0}=\chi\left(\mathbf{x}_{0}, t\right)-\mathbf{x}_{0} .
$$

Spatial derivatives of the motion function $\chi\left(\mathbf{x}_{0}, t\right)$ in the Lagrangian description can be used to calculate the deformation gradient $\mathbf{F}$ :

$$
\mathbf{F}=\operatorname{Grad} \boldsymbol{\chi}\left(\mathbf{x}_{0}, t\right)=\operatorname{Grad} \mathbf{u}\left(\mathbf{x}_{0}, t\right)+\mathbf{I},
$$

where I represents the second order identity tensor and the operator Grad is the gradient performed in the reference configuration. Basically, $\mathbf{F}$ is not the 
gradient of a deformation, since it also contains rigid rotations. The polar decomposition allows to separate the rotation, indicated by the tensor $\mathbf{R}$, from pure deformation, indicated by the right and left stretch tensors, i.e. $\mathbf{U}$ and $\mathbf{V}$ respectively:

$$
\mathbf{F}=\mathbf{R U}=\mathbf{V R}
$$

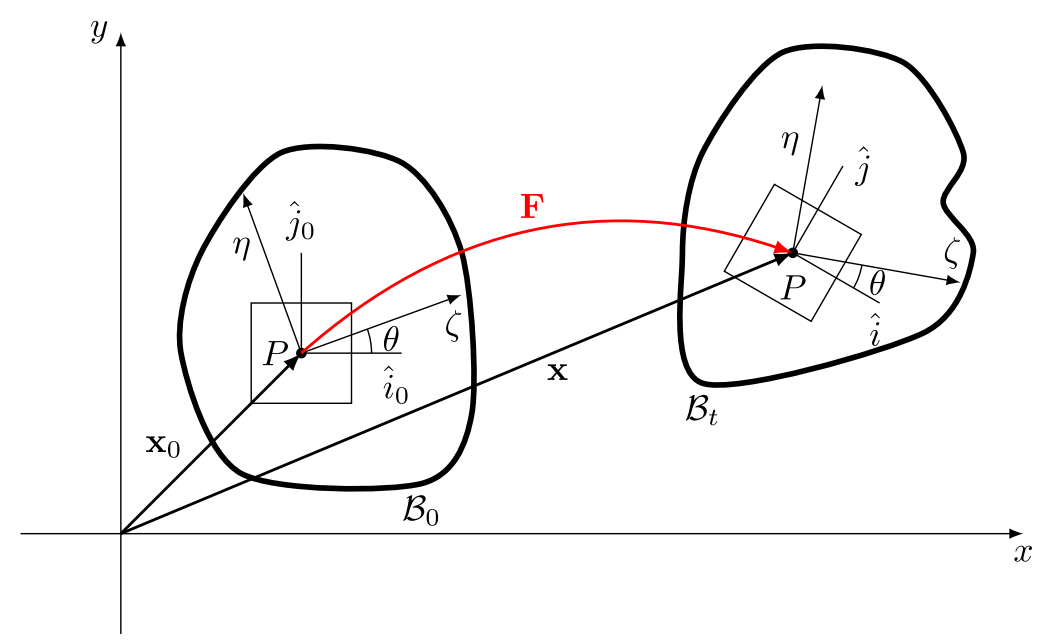

Figure 1: Kinematic of material body at finite strains with the definition of reference and current placement and including the material orientation $\theta$.

The VFM relies on the Principle of Virtual Work (PVW) that, in finite deformation theory, can be written either in the reference or current placement. So, using the Lagrangian description and neglecting body forces and inertia (quasi-static loading), the PVW can be written as:

$$
\int_{\mathcal{B}_{0}} \mathbf{T}_{1 P K}: \delta \mathbf{F}^{*} d V_{0}=\int_{\partial \mathcal{B}_{0}}\left(\mathbf{T}_{1 P K} \hat{\mathbf{n}}_{0}\right) \cdot \delta \mathbf{u}^{*} d A_{0},
$$

where $\delta \mathbf{u}^{*}$ is an arbitrary kinematically admissible virtual displacement field, 
$\delta \mathbf{F}^{*}$ the corresponding deformation gradient and $\mathbf{T}_{1 P K}$ the First Piola-Kirchhoff stress tensor, which is obtained as:

$$
\mathbf{T}_{1 P K}=\operatorname{det}(\mathbf{F}) \boldsymbol{\sigma} \mathbf{F}^{-T}
$$

where $\boldsymbol{\sigma}$ is the Cauchy stress tensor. The first therm of Eq. 4 is the internal Virtual Work (IVW) that must be equal to the external Virtual Work (EVW), i.e. the second therm of Eq. 4.

Introducing a generic constitutive law for plasticity, which expresses the stress as a function of the deformation history $\mathbf{F}(t)$, regulated by a set of $m$ parameters $\boldsymbol{\xi}=\left[\xi_{1}, \xi_{2}, \ldots, \xi_{m}\right]$, for each material point, the stress tensor $\boldsymbol{\sigma}=\boldsymbol{\sigma}(\mathbf{F}(t), \boldsymbol{\xi})$. Thereby, if the deformation history of the body $\mathcal{B}_{0}$ is known, the following cost function can be defined:

$$
\psi\left(\boldsymbol{\xi}, \delta \mathbf{u}^{*}, t\right)=\left|\int_{\mathcal{B}_{0}} \mathbf{T}_{1 P K}: \delta \mathbf{F}^{*} d V_{0}-\int_{\partial \mathcal{B}_{0}}\left(\mathbf{T}_{1 P K} \hat{\mathbf{n}}_{0}\right) \cdot \delta \boldsymbol{u}^{*} d A_{0}\right| .
$$

If the constitutive parameters $\boldsymbol{\xi}$ are exact, the cost function $\psi$ must be equal to zero for any load step $t$ of the test and for any selected virtual fields (VF) $\delta \boldsymbol{u}^{*}$. Moreover, when the VFM is applied to full-field measurement data, the region of interest (ROI) considered is represented by a regularly distributed grid of $n_{P}$ measurement points, and the IVW integral can be numerically solved as a discrete sum. In the case of flat specimens with constant thickness $t_{0}$, Eq. 6 becomes:

$$
\psi\left(\boldsymbol{\xi}, \delta \mathbf{u}^{*}, t\right)=\left|\sum_{w=1}^{n_{P}}\left(\mathbf{T}_{1 P K}^{(w)}: \delta \mathbf{F}^{*(w)} S^{(w)} t_{0}\right)-\int_{\partial \mathcal{B}_{0}}\left(\mathbf{T}_{1 P K} \hat{\mathbf{n}}_{0}\right) \cdot \delta \boldsymbol{u}^{*} d A_{0}\right|
$$


where $S^{(w)}$ is the surface area covered by the $w$-th measurement point.

If we consider $n_{t}$ different tests, with $n_{s}$ time steps and $n_{v f} \mathrm{VFs}$, a global cost function can be defined as follows:

$$
\Psi(\boldsymbol{\xi})=\sum_{i=1}^{n_{t}} \sum_{j=1}^{n_{v f}} \sum_{k=1}^{n_{s}} \psi_{i}\left(\boldsymbol{\xi}, \delta \mathbf{u}_{j}^{*}, t_{k}\right) .
$$

In non-linear problems, the constitutive parameters $\boldsymbol{\xi}$ are identified as those minimizing the function $\Psi(\boldsymbol{\xi})$.

Often, gathering displacement and strain data from the bulk of the specimen is not a straightforward process, and only full-field data measured at its surface are used for the VFM. This leads to assume a constant distribution of the mechanical fields in the trough-thickness direction; so, since the identification is performed on specimens obtained from metal sheets, the plane stress condition is assumed due to the small thickness compared to the other two dimensions. In this way, all the out-of-plane components of the stress tensor are neglected $\sigma_{33}=\sigma_{13}=\sigma_{23}=0$, and, also, only the components of $\delta \mathbf{F}^{*}$ which effectively contribute to the IVW are defined.

\subsection{Stress calculation from strain fields}

At each iteration of the minimization process, the evaluation of the VFM cost function in Eq. 66 requires the calculation of the $\mathbf{T}_{1 P K}$ tensor. However, constitutive equations are generally defined in the material coordinate system $(\zeta, \eta)$, as depicted in Figure 1, that, in anisotropic plasticity problems, is aligned with the material texture.

According to the incremental deformation theory based on minimum plastic work path (proportional logarithmic strain path) [39], the strain increment 
is expressed by using the spatial logarithmic strain definition $\varepsilon$ (also called Hencky strain) on the left stretch tensor $\mathbf{V}$, i.e.:

$$
\varepsilon=\ln \mathbf{V}
$$

where $\mathbf{V}$ is computed as:

$$
\mathbf{V}=\sqrt{\mathbf{F F}^{T}}
$$

This kinematic quantity is computed in the global coordinate system $\left(\hat{i}_{0}, \hat{j}_{0}\right)$; thus, the strain fields must be projected to the material coordinate system:

$$
\boldsymbol{\varepsilon}_{\text {mat }}=\mathbf{R}_{\text {mat }}^{T}\left(\mathbf{R}^{T} \boldsymbol{\varepsilon} \mathbf{R}\right) \mathbf{R}_{\text {mat }}
$$

where $\mathbf{R}_{\text {mat }}$ indicates the rotation tensor to rotate the global coordinate system into the material one in the reference placement. Thus, according to additive decomposition of the strain, the elastic strain increment is decoupled from the plastic one as follows:

$$
\Delta \varepsilon_{m a t}=\Delta \varepsilon_{m a t}^{e}+\Delta \varepsilon_{m a t}^{p}
$$

Generally, for inverse problems, the Cauchy stress tensor is calculated via implicit algorithms based on the elastic predictor - plastic corrector scheme [25, 30, 33, 40]. However, such algorithms may suffer from convergence problems when the strain increment is too large [41], and usually require a large number of iterations to solve the set of non-linear equations describing the material plastic behavior. Here, under the assumption of associated flow rule, the stress integration is achieved with the direct method introduced by 
Rossi and Pierron in [29], capable of reconstructing the stress fields with no iterations even in case of large strain increment. However, a complete definition of the direct method is beyond the scope of this paper, and a detailed explanation of the algorithm is reported in [31] with its application to Hill48 and Yld2000-2D anisotropic models.

The Cauchy stress tensor $\boldsymbol{\sigma}_{m a t}$ is calculated from $\boldsymbol{\varepsilon}_{m a t}$ and defined in the material frame. Finally, the $\boldsymbol{\sigma}_{m a t}$ tensor is - then - rotated to the global coordinate system:

$$
\boldsymbol{\sigma}=\mathbf{R}_{m a t}\left(\mathbf{R} \boldsymbol{\sigma}_{m a t} \mathbf{R}^{T}\right) \mathbf{R}_{m a t}^{T} ;
$$

this expression is introduced in Eq. 5 to obtain the first Piola-Kirchhoff tensor $\mathbf{T}_{1 P K}$.

\subsection{Definition of Virtual Fields}

The PVW expressed in Eq. 44 is valid for any VFs kinematically coherent with the problem. However, their choice affects the identification results, because they provide different weights to the material points included in the cost function. Two approaches for virtual fields selection can be distinguished according to their generation method. The first approach is based on user defined virtual fields (UDVFs), employing polynomial or harmonic functions that usually relies on the user experience. This operation is not always trivial and needs a deep understanding of the problem. For instance, in [30] six different virtual fields were introduced for the identification of anisotropic plasticity constitutive coefficients, and their performances were evaluated with a competitive comparison on numerical data. Another example can be 
found in [31, where three empirical virtual fields were selected in order to activate different stress components. In this work, the same virtual fields were employed in the identification for the UDVFs approach. Therefore, adopting the coordinate system in Figure 2, the three VFs and the corresponding gradients are, respectively:

$$
\begin{aligned}
& \left\{\begin{array} { l } 
{ \delta u _ { x } ^ { * ( 1 ) } = 0 } \\
{ \delta u _ { y } ^ { * ( 1 ) } = \frac { y } { h } }
\end{array} \quad \left\{\begin{array}{l}
\delta u_{x}^{*(2)}=\frac{x}{w} \frac{|y|-h}{h} \\
\delta u_{y}^{*(2)}=0
\end{array}\right.\right. \\
& \left\{\begin{array}{l}
\delta u_{x}^{*(3)}=\frac{1}{\pi} \sin \left(\pi \frac{x}{w}\right) \cos \left(\pi \frac{y}{2 h}\right) \\
\delta u_{y}^{*(3)}=\frac{1}{\pi} \sin \left(\pi \frac{x}{w}\right) \cos \left(\pi \frac{y}{2 h}\right)
\end{array}\right.
\end{aligned}
$$

and:

$$
\begin{aligned}
& \left\{\begin{array} { l } 
{ \delta \mathbf { F } _ { x x } ^ { * ( 1 ) } = \delta \mathbf { F } _ { x y } ^ { * ( 1 ) } = \delta \mathbf { F } _ { y x } ^ { * ( 1 ) } = 0 } \\
{ \delta \mathbf { F } _ { y y } ^ { * ( 1 ) } = \frac { 1 } { h } }
\end{array} \quad \left\{\begin{array}{l}
\delta \mathbf{F}_{x x}^{*(2)}=\frac{|y|-h}{w h} \\
\delta \mathbf{F}_{x y}^{*(2)}=\operatorname{sgn}(y) \frac{x}{w h} \\
\delta \mathbf{F}_{y x}^{*(2)}=\delta \mathbf{F}_{y y}^{*(2)}=0
\end{array}\right.\right. \\
& \left\{\begin{array}{l}
\delta \mathbf{F}_{x x}^{*(3)}=\delta \mathbf{F}_{y x}^{*(3)}=\frac{1}{w} \cos \left(\pi \frac{x}{w}\right) \cos \left(\pi \frac{y}{2 h}\right) \\
\delta \mathbf{F}_{x y}^{*(3)}=\delta \mathbf{F}_{y y}^{*(3)}=-\frac{1}{2 h} \sin \left(\pi \frac{x}{w}\right) \sin \left(\pi \frac{y}{2 h}\right)
\end{array}\right.
\end{aligned}
$$

It is also worth noting that the first set of VFs in Eq. 14 makes the EVW proportional to the measured loading force $\int_{\partial \mathcal{B}_{0}}\left(\mathbf{T}_{1 P K} \hat{\mathbf{n}}_{0}\right) \cdot \delta \mathbf{u}^{*} d A_{0}=2 F$, and affects only the stress component in the vertical direction. For the other two sets of VFs, the EVW is equal to zero. 


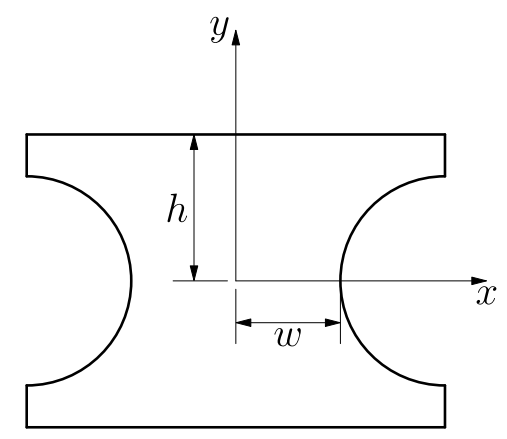

Figure 2: Schematic of the reference frame adopted for the virtual fields definition.

The second approach for virtual fields selection is based, instead, on their automatic generation according to suitable criteria. This characteristic, in fact, reduces the dependence of user knowledge for the identification procedure setting, making the VFM more attractive for industrial applications. A first implementation can be found in [35] for the case of linear anisotropic elasticity, where the procedure for virtual fields definition is governed by the minimization of noise effects on the identified constitutive coefficients. Lately, such procedure was extended to isotropic elasto-plasticity problems [33, demonstrating that the implementation of optimized virtual fields is capable of improving the results accuracy. A recent contribution to automatic virtual fields approach for non-linear constitutive models was proposed by Marek et al. [42] in the case of isotropic plasticity and, then, extended to anisotropy models like Hill48 and Yld2000 in [36]. Here, the generation criterion relies on the sensitivity of the reconstructed stress field to each parameter of the constitutive model. Considering a set of parameters $\boldsymbol{\xi}$, the stress sensitivity maps corresponding to the parameter $\xi_{j}$ can be generated as: 


$$
\delta \mathbf{T}_{1 P K}^{(j)}(\boldsymbol{\xi}, t)=\mathbf{T}_{1 P K}\left(\boldsymbol{\xi}-\delta \xi_{j}, t\right)-\mathbf{T}_{1 P K}(\boldsymbol{\xi}, t),
$$

where $\delta \xi_{j}$ indicates a perturbation of the parameter $\xi_{j}$ and, generally, $0.10 \xi_{j} \leq$ $\delta \xi_{j} \leq 0.20 \xi_{j}$. The stress sensitivity can also be expressed in an incremental form, as:

$$
\delta \tilde{\mathbf{T}}_{1 P K}^{(j)}(\boldsymbol{\xi}, t)=\frac{\delta \mathbf{T}_{1 P K}^{(j)}(\boldsymbol{\xi}, t)-\delta \mathbf{T}_{1 P K}^{(j)}(\boldsymbol{\xi}, t-\Delta t)}{\Delta t} .
$$

Such sensitivity maps are used to generate virtual strain fields, amplifying the information related to each constitutive parameter in the VFM cost function. This problem is tackled following a finite element approach where the domain is subdivided in a virtual mesh using 4-nodes quadrilateral elements, according to the piecewise virtual field implementation in [27]. Afterwards, it is possible to define a set of virtual nodal displacements whose derivation produces a virtual strain fields similar to the stress sensitivity maps. In particular, the virtual nodal displacements $\delta \mathbf{u}_{N}^{*}$ are linked to the virtual deformation gradient field using the element shape functions, so:

$$
\delta \mathbf{F}^{*}=\mathbf{B} \delta \mathbf{u}_{N}^{*},
$$

where the matrix $\mathbf{B}$ stands for the global strain-displacement matrix relating virtual displacements to their spatial gradients. Moreover, the global strain-displacement matrix must be corrected in order to ensure the particular boundary conditions of the problem, constraining the displacements of the corresponding nodes. Consequently, the virtual displacements are calculated from the stress sensitivity maps employing the pseudoinverse of the modified global strain-displacement matrix $\overline{\mathbf{B}}$, so that: 


$$
\delta \mathbf{u}_{N}^{*}(\boldsymbol{\xi}, t)=\operatorname{pinv}(\overline{\mathbf{B}}) \delta \mathbf{T}_{1 P K}(\boldsymbol{\xi}, t),
$$

where the $\operatorname{pinv}(\cdot)$ operator indicates the Moore-Penrose pseudoinverse matrix, computed numerically by means of singular value decomposition.

Once the nodal virtual displacement are determined, the so called sensitivity based virtual fields (SBVFs) are obtained. Further details about the procedure for SBVFs generation are described in [36].

\subsection{Constitutive model: the Yld2000-2D}

The Yld2000-2D yielding function [6] describes the anisotropic behaviour of materials by means of two functions of linear transformations of the deviatoric Cauchy stress tensor $\mathbf{s}$. The yield function $\phi$ is defined as:

$$
\phi=\phi^{\prime}+\phi^{\prime \prime}=2 \bar{\sigma}^{a},
$$

where $\bar{\sigma}$ indicates the equivalent stress and the two functions are expressed as:

$$
\begin{aligned}
& \phi^{\prime}=\left|X_{1}^{\prime}-X_{2}^{\prime}\right|^{a} \\
& \phi^{\prime \prime}=\left|2 X_{2}^{\prime \prime}+X_{1}^{\prime \prime}\right|^{a}+\left|2 X_{1}^{\prime \prime}+X_{2}^{\prime \prime}\right|^{a} .
\end{aligned}
$$

In particular, the exponent $a$ depends on the crystal structure of the material, and controls the curvature of yield surface vertices, ensuring its convexity when $a \geq 1$. Usually the $a$ value is set equal to 6 in case of BCC materials or 8 for FCC materials [43] and is not calibrated from the experiments. 
Observing the two functions, the $X_{j}^{\prime}$ and $X_{j}^{\prime \prime}$ (with $j=1,2$ ) represent the principal values of $\mathbf{X}^{\prime}$ and $\mathbf{X}^{\prime \prime}$ tensors respectively. Indicating with $x$ the rolling direction (RD) and $y$ the transverse direction (TD), such tensors are defined as linear transformation of the stress deviator:

$$
\left\{\begin{array}{l}
X_{x x}^{\prime} \\
X_{y y}^{\prime} \\
X_{x y}^{\prime}
\end{array}\right\}=\left[\begin{array}{ccc}
C_{11}^{\prime} & C_{12}^{\prime} & 0 \\
C_{21}^{\prime} & C_{22}^{\prime} & 0 \\
0 & 0 & C_{33}^{\prime}
\end{array}\right]\left\{\begin{array}{l}
s_{x x} \\
s_{y y} \\
s_{x y}
\end{array}\right\}
$$

and

$$
\left\{\begin{array}{l}
X_{x x}^{\prime \prime} \\
X_{y y}^{\prime \prime} \\
X_{x y}^{\prime \prime}
\end{array}\right\}=\left[\begin{array}{ccc}
C_{11}^{\prime \prime} & C_{12}^{\prime \prime} & 0 \\
C_{21}^{\prime \prime} & C_{22}^{\prime \prime} & 0 \\
0 & 0 & C_{33}^{\prime \prime}
\end{array}\right]\left\{\begin{array}{l}
s_{x x} \\
s_{y y} \\
s_{x y}
\end{array}\right\}
$$

Moreover, for simplicity, these equations can be extended to the Cauchy stress tensor $\boldsymbol{\sigma}$ through $\mathbf{T}$ :

$$
\begin{aligned}
& X^{\prime}=\mathbf{C}^{\prime} s=\mathbf{C}^{\prime} \mathbf{T} \boldsymbol{\sigma}=\mathbf{L}^{\prime} \boldsymbol{\sigma} \\
& X^{\prime \prime}=\mathrm{C}^{\prime \prime} s=\mathrm{C}^{\prime \prime} \mathbf{T} \boldsymbol{\sigma}=\mathbf{L}^{\prime \prime} \boldsymbol{\sigma},
\end{aligned}
$$

with:

$$
\mathbf{T}=\left[\begin{array}{ccc}
\frac{2}{3} & -\frac{1}{3} & 0 \\
-\frac{1}{3} & \frac{2}{3} & 0 \\
0 & 0 & 1
\end{array}\right]
$$

The two tensors $\mathbf{L}^{\prime}$ and $\mathbf{L}^{\prime \prime}$ are function of eight independent coefficients $\alpha_{i}:$ 


$$
\begin{aligned}
& \mathbf{L}^{\prime}=\frac{1}{3}\left[\begin{array}{ccc}
2 \alpha_{1} & -2 \alpha_{1} & 0 \\
2 \alpha_{2} & 2 \alpha_{2} & 0 \\
0 & 0 & 3 \alpha_{7}
\end{array}\right] \\
& \mathbf{L}^{\prime \prime}=\frac{1}{9}\left[\begin{array}{ccc}
8 \alpha_{5}-2 \alpha_{3}-2 \alpha_{6}+2 \alpha_{4} & -4 \alpha_{6}-4 \alpha_{4}-4 \alpha_{5}+\alpha_{3} & 0 \\
-4 \alpha_{3}-4 \alpha_{5}-4 \alpha_{4}+\alpha_{6} & 8 \alpha_{4}-2 \alpha_{6}-2 \alpha_{3}+2 \alpha_{5} & 0 \\
0 & 0 & 9 \alpha_{8}
\end{array}\right]
\end{aligned}
$$

The standard calibration of the eight coefficients $\alpha$ of the Yld2000-2D yield surface generally requires experimental data in terms of flow stresses and the width-to-thickness strain ratio ( $r$-value, also known as Lankford coefficient), obtained from uniaxial and balanced biaxial stress states; so, the yield function coefficients are retrieved by using a non-linear NewtonRaphson solver, as reported in the Appendix of [6]. Similarly, in this paper the identification process through the VFM was restricted to the characterization of the anisotropy coefficients $\alpha$, while the $a$ exponent was selected according to the material crystal structure. The identification of the material parameter $a$ from real experiments is nonetheless an interesting topic that could be investigated in future studies, using suitably designed specimens.

\section{Inverse identification strategy}

The inverse identification protocol adopted in this study is depicted in Figure 3. The input data are the geometry of the specimens, the displacement fields measured with DIC and the force measured by the load cell of the tensile machine. Afterwards, the strain field is computed using Eq. 9, 10 and 11 , with temporal and spatial smoothing to reduce the experimental error. 
As a first step, the hardening parameters are identified using the linear stress-strain curve identification (LSSCI) method, described in [37]. Starting from heterogeneous full-field data, the LSSCI retrives the flow curve in terms of a piecewise function, using a pseudo-inverse function, with a relevant computational efficiency. This procedure permits to include material information in the post-necking regime, and, also, can be employed to assess the quality of submitted strain field data, helping the user to evaluate an adequate smoothing grade.

\begin{tabular}{|l|}
\hline Input data: \\
- Geometry \\
- Displacement fields $\left(u_{x}, u_{y}\right)$ \\
- Measured force $P$ \\
\hline
\end{tabular}
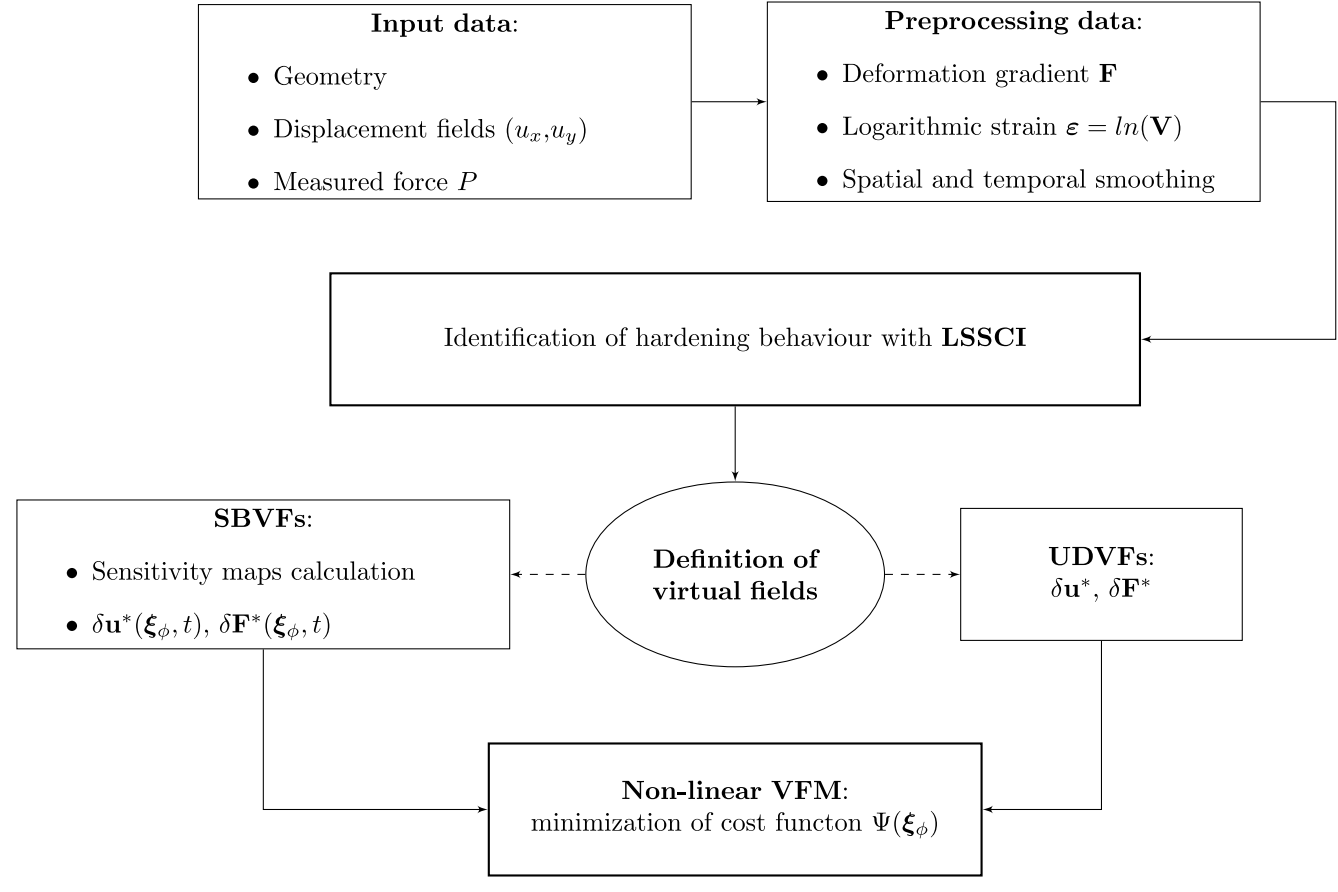

Figure 3: Flowchart of the proposed identification procedure.

The application of non-linear VFM is restricted, then, only to the identification of anisotropic yield function coefficients, which are iteratively changed by the optimization algorithm until the VFM cost function in Eq. 8 is mini- 
mized. Excluding the hardening parameters from the VFM abets its convergence, reducing significantly the computational effort for the identification. The implemented procedure allows to select the two different types of virtual fields previously discussed, choosing between the UDVFs and SBVFs. Eq. 19 indicates that the SBVFs are computed for every time step at the beginning of each iteration performed by the minimization algorithm. The identification framework presented in [36] includes a selective updating algorithm that, basically, generates a new set of SBVFs until the variation of the VFM cost function $\Delta \Psi$ between two consecutive iterations is below a certain tolerance. In this study, in order to enhance the computational efficiency of the whole non-linear VFM procedure, the SBVFs construction occurs only at the beginning of the identification process employing the initial guess parameters, without any upgrade during the minimization. Moreover, since all the target coefficients of the anisotropic yield function are set equal to 1 at the initial iteration, the corresponding SBVFs are built from the stress sensitivity obtained perturbing the isotropic case.

Marek et al. in [36] highlight how the minimization method can also affect the computational efficiency, comparing two widely diffused optimization algorithms: the Sequential Quadratic Programming (SQP) and the LevenbergMarquardt algorithm (LMA). In this paper, all the identification results for the involved data sets are obtained adopting the latter; in fact, although its accuracy is strongly affected by the initial guess of the target parameters, the LMA offers an higher computational efficiency compared to the SQP, achieving convergence with a reduced number of iterations. More non-linear optimization methods have been tested in [44. 


\section{Numerical Validation}

The feasibility of the presented identification strategy was firstly evaluated on numerical data. The best specimen shape to be used in inverse identification is still an open issue [45, 46]; for example, in [21], a double perforated specimen was used to identify the 3D Hill48 yield function, Kim et al. [30] proposed a $\Sigma$-shaped specimen, etc. Here, following the approach used in [31, 36], tensile tests on deep notched specimens were employed. The presence of circular notches, in fact, produces an heterogeneous strain field on the specimen surface with a diffused plastic zone [22], permitting to feed the identification with a larger number of information compared to the standard unaxial test.

The tensile tests were reproduced with ABAQUS-Standard ${ }^{\circledR}$, using CPS4 4 -nodes elements and considering three different material orientations $\left(0^{\circ}\right.$, $\left.45^{\circ}, 90^{\circ}\right)$. Figure 4 illustrates the FEM mesh as well as the dimension of the specimen.

The hardening behaviour was described by the Swift Law:

$$
\bar{\sigma}=K\left(\varepsilon_{0}+\bar{\varepsilon}_{p}\right)^{N}
$$

where $K, \varepsilon_{0}$ and $N$ are the model material coefficients, and $\bar{\varepsilon}_{p}$ indicates the accumulated equivalent plastic strain, defined incrementally through the plastic work equivalence $\bar{\sigma} d \bar{\varepsilon}_{p}=\boldsymbol{\sigma}: d \boldsymbol{\varepsilon}$. The Yld2000-2D anisotropy model was implemented with the UMAT subroutine following the stress integration scheme given by [9, 39]. All reference material coefficients adopted are listed in Table 1 .

A region of interest (ROI) was considered in the specimen's area between 
Table 1: Reference material parameters for the hardening law and anisotropic yield function adopted in the FE model.

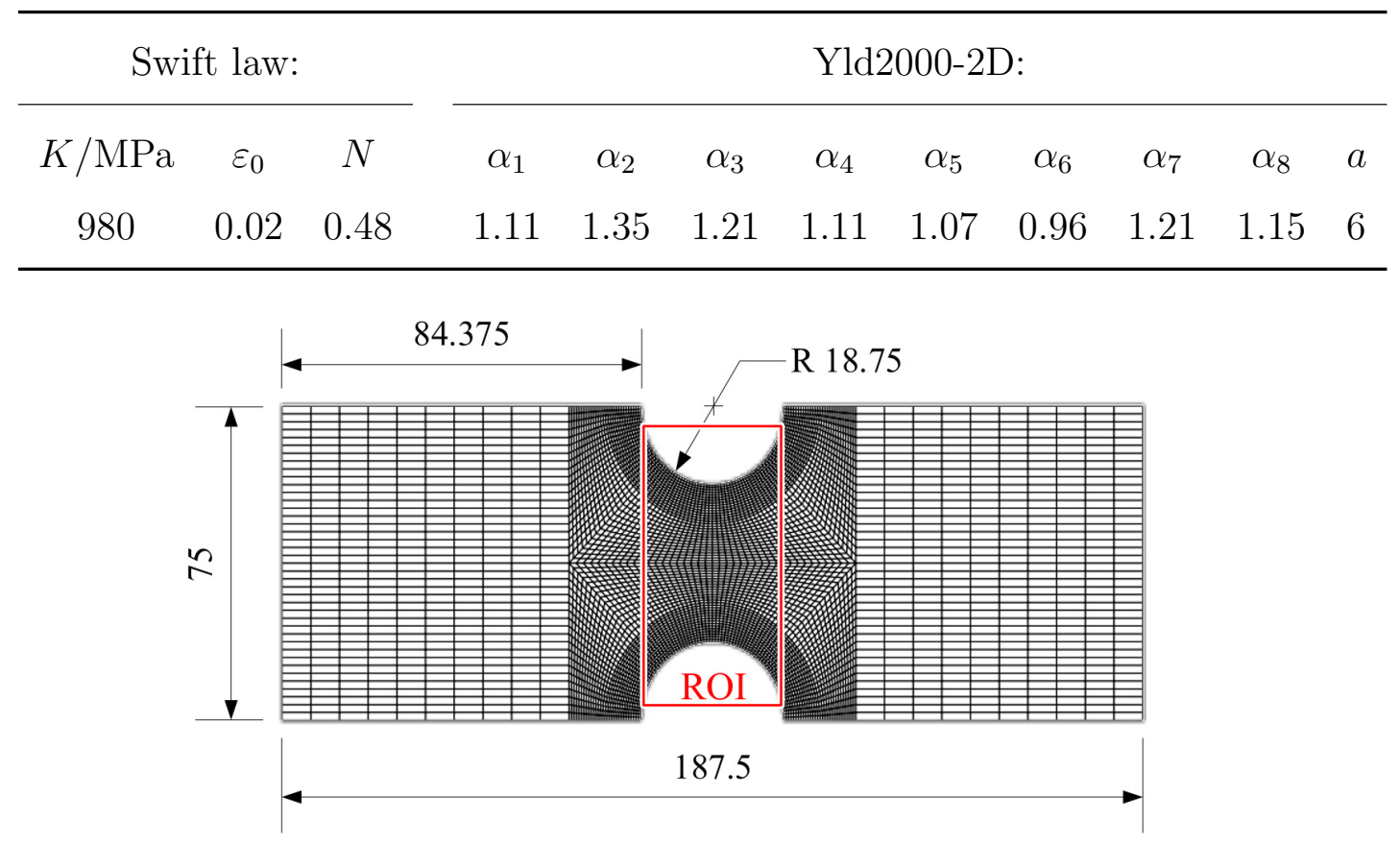

Figure 4: Notched specimen geometry adopted for the numerical validation, the units are in $\mathrm{mm}$.

the two notches, as highlighted in Figure 4. Within the ROI, to simulate an actual DIC measurement, a set of $141 \times 281$ equi-spaced displacement points was generated by interpolation from the FE results. The loading path was subdivided into 25 time steps. The full decoupled identification scheme was applied on numerical data including two different data sets in the non-linear VFM process: the first composed by strain data from specimens cut at three different material orientations, the second composed only by one specimen oriented at $45^{\circ}$ with respect to the $\mathrm{RD}$.

Since the physical meaning of the Yld2000-2D coefficients is not straight- 




Figure 5: Prediction of uniaxial flow stresses and $r$-values by using different VFs and numerical data sets.

forward, an effective way to visualize the accuracy of the identification results is given by their capability to predict the anisotropy of normalized flow stress and $r$-values at different material orientations [47] (Figure 5). Furthermore, the flow stress was normalized using the equi-biaxial stress, in order to indirectly include in the chart information about such stress state. The non-linear VFM was applied using both UDVFs and SBVFs, the latter constructed adopting a virtual mesh of $20 \times 20$ elements and a perturbation parameter $\delta \xi_{j}=0.15$. In the graphs, the identification with UDVFs is denoted by lines with dots while SBVFs with plain lines. The same approach of Figure 5 was used in the rest of the paper to analyze the identification results, however, for completeness, all numerical coefficients obtained in the different identifications were listed in the Appendix.

The outcomes can be summarized as follows:

- the Yld2000-2D's $\alpha$-coefficients identified exploiting the tests at three 
material orientations produces an accurate identification of the anisotropic behaviour, indicating that a rich data set helps to constrain the minimization process. For the normalized stress, the average error between the reference curve and identified one is around $1 \%$ for both UDVFs and SBVFs. For the $r$-values, the error using SBVFs is around 1-2\% while it slightly increase using UDVFs, i.e around $2-4 \%$.

- When the proposed scheme is applied only to data from the specimen oriented at $45^{\circ}$, the UDVFs are not capable of capturing correctly the anisotropic behaviour of the material, giving totally inaccurate results and confirming the conclusions of [36]. Using SBVFs, instead, the identified parameters are able to predict the general trend, but a shift is observed with respect to the reference curves in both normalized stress and $r$-value. The shift in the normalized stress indicates that the biaxial stress was not identified properly.

According to the identification strategy proposed in this paper, the hardening curve was identified before the non-linear VFM using the LSSCI method, as previously illustrated in the flowchart of Figure 3. The LSSCI method was applied on the specimen at $0^{\circ}$, using a piecewise function with 11 divisions and without introducing the material anisotropy (LSSCI, von Mises). Then, the non-linear VFM was employed to identify the anisotropy coefficients.

Figure 6 shows the comparison of the flow curves corresponding to $0^{\circ}$ orientation at the end of the identification procedure. Interestingly, although the hardening behaviour was not updated during the non-linear VFM process, a good agreement is found between the reference curve and the piecewise curve identified initially with LSSCI and von Mises. As countercheck, the 


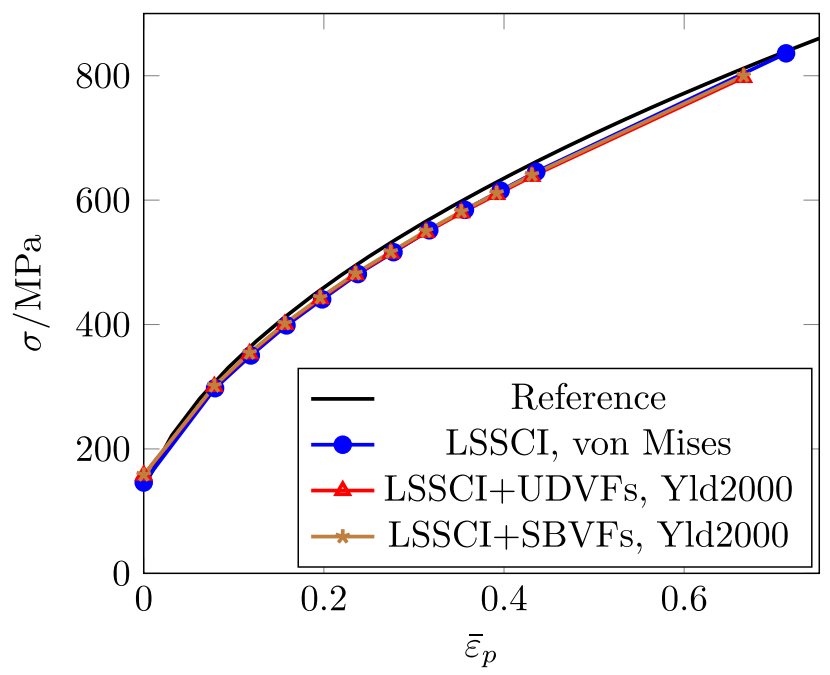

Figure 6: Comparison of identified flow stress curves at $0^{\circ}$ orientation.

LSCCI was applied again employing the identified Yld2000-2D parameters and, as illustrated in Figure 6, there are not significant differences between the curves.

From the numerical validation, it turns out that the identification strategy proposed in this paper allows to identify correctly the coefficients of Yld2000-2D if three tests on three directions are used.

\section{Experiments on BH340 steel}

In the following sections, the identification procedure presented in this paper was applied to characterize the Yld2000-2D model for the BH340 steel. First, the anisotropic model was calibrated using the standard protocol described in [6], with the aim to provide a touchstone for the evaluation of the identification results. Then, the inverse identification was performed using tensile tests on notched specimens. The hardening behaviour was identified 
by LSSCI and the non-linear VFM was used to characterize the anisotropic yield function.

The elastic properties for this material are known, Young Modulus $E=$ $210 \mathrm{GPa}$ and Poisson's Ratio $\nu=0.3$. Since the BH340 has a BCC crystal structure, the material exponent $a$ was imposed equal to 6 , making the VFM minimization applied only to the eight $\alpha_{j}$ coefficients of Yld2000-2D.

\subsection{Standard Calibration}

According to the standard calibration protocol of the Yld2000-2D material model, uniaxial tension test along the RD and TD and the equibiaxial stress state provide three flow stresses $\sigma_{0}, \sigma_{90}, \sigma_{B}$ and three $r$-values $r_{0}, r_{90}$, $r_{B}$, which are necessary to calibrate the coefficients from $\alpha_{1}$ to $\alpha_{6}$. It is worth noting that $r_{B}$ represents the slope of the yield surface at balanced biaxial stress state, and is defined as $r_{B}=\dot{\varepsilon}_{y y} / \dot{\varepsilon}_{x x}$. The flow stress and $r$ value obtained from uniaxial tensile test at $45^{\circ}$ from RD supply the remaining coefficients $\alpha_{7}$ and $\alpha_{8}$. In order to have a complete insight of material anisotropic behaviour, standard uniaxial flat specimens with seven different directions from the RD were machined from the same $0.7 \mathrm{~mm}$ thick BH340 sheet metal and tested. Displacement and strain fields on the specimen surface were retrieved using a stereo-DIC set-up composed of two $2448 \times 2048$ CCD cameras, synchronized with the $500 \mathrm{kN}$ loading cell to couple each frame with the measured force (Figure 7a). The commercial software MatchID $^{\circledR}$ (www.matchid.eu, version 2018.2.2) was employed to perform the measurements. All the details about the setting used for DIC analysis are reported in Appendix B under Table 8, and the engineering curves resulting from the quasi-static tests (at $\dot{\varepsilon}=0.01 s^{-1}$ ) are reported in Figure $7 \mathrm{~b}$. The engineering 


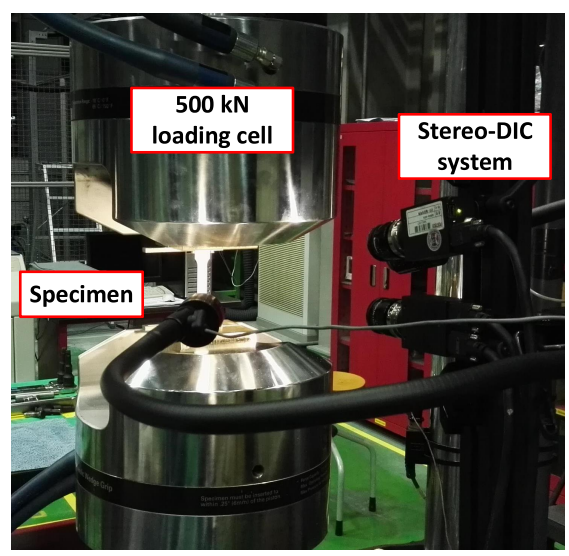

(a)

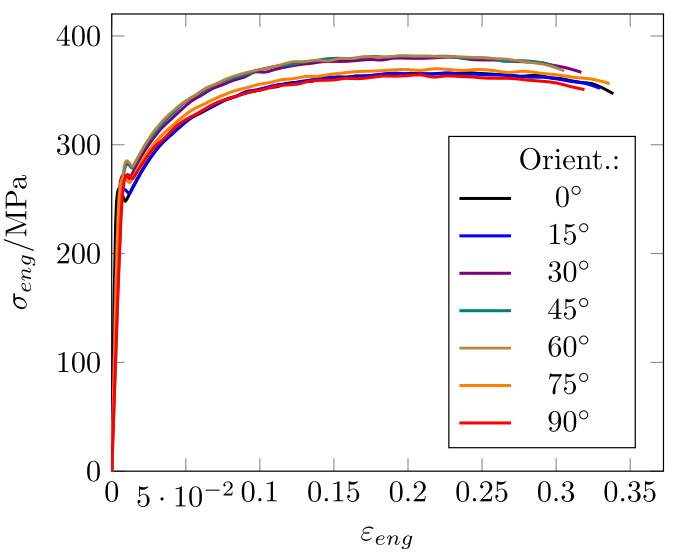

(b)

Figure 7: Uniaxial test experimental setup (a). Engineering stress-strain curves obtained at seven different directions from the RD (b).

curves show an higher flow stress at $30^{\circ}, 45^{\circ}$ and $60^{\circ}$.

The equibiaxial stress state condition was obtained by means of a hydraulic bulge test [48, 49]. According to the ISO 16808:2014 standard [50], the calculation of the biaxial stress-strain curve requires the measurement of three quantities: the forming pressure of the fluid, the bulge curvature, and the through-thickness strain. Full-field measurements with stereo-DIC allows the determination of the latter two inputs; the in-plane strains can be derived from displacements fields, and, then, the through-thickness strain component from the hypothesis of incompressibility. The used stereo-DIC system is illustrated in Figure 8 together with the strain fields measured at last step along the RD and TD,. The analysis parameters of the correlation software $M a t c h I D^{\circledR}$ are listed in Table 9. The results are reported in Figure 9, where the $r$-value relative to the biaxial stress state $r_{B}$ is calculated by means of a linear regression of the RD vs. TD strain data points. 


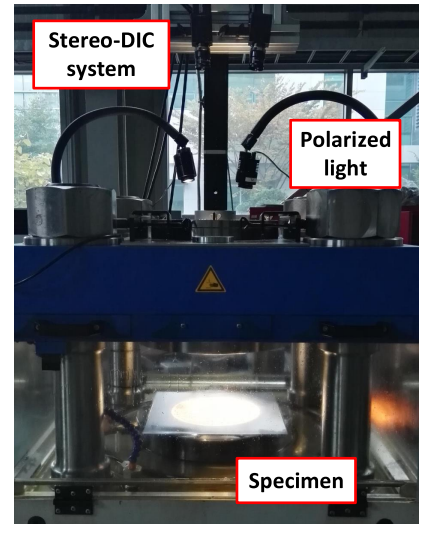

(a)

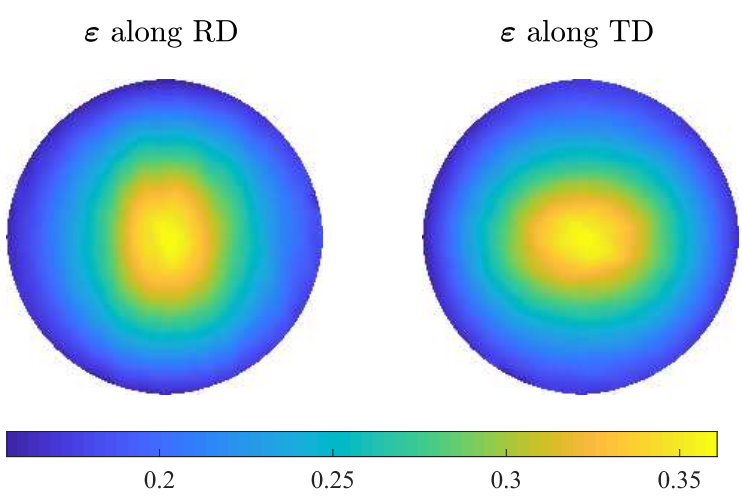

(b)

Figure 8: Hydraulic bulge test machine with DIC set-up (a). Logarithmic strain fields along the RD and TD directions (b).

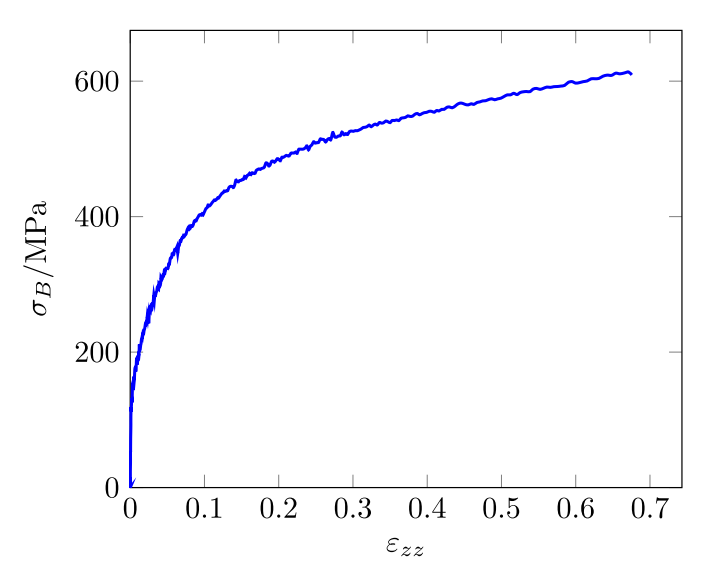

(a)

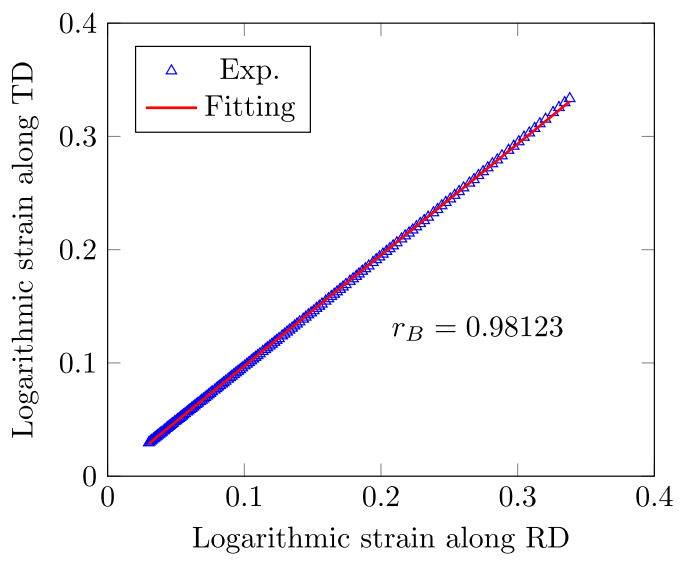

(b)

Figure 9: Hydraulic bulge test results for BH340: (a) logarithmic stress-strain curve in balanced biaxial loading, where $\varepsilon_{z z}$ indicates the through-thickness strain; (b) calculation of biaxial $r$-value. 
Table 2: Input data for Yld2000-2D calibration and results.

\begin{tabular}{|c|c|c|c|c|c|c|c|c|}
\hline \multicolumn{9}{|c|}{ Input experimental data: } \\
\hline Dir. & $0^{\circ}$ & $15^{\circ}$ & $30^{\circ}$ & $45^{\circ}$ & $60^{\circ}$ & $75^{\circ}$ & $90^{\circ}$ & Biax. \\
\hline$\sigma / \sigma_{B}$ & 0.9219 & 0.9180 & 0.9508 & 0.9558 & 0.9567 & 0.9288 & 0.9154 & 1 \\
\hline$r$ & 1.52 & 1.30 & 1.00 & 0.89 & 1.06 & 1.48 & 1.67 & 0.98 \\
\hline \multicolumn{9}{|c|}{ Parameters calibrated with the standard procedure: } \\
\hline$\alpha_{1}$ & $\alpha_{2}$ & $\alpha_{3}$ & $\alpha_{4}$ & $\alpha_{5}$ & $\alpha_{6}$ & $\alpha_{7}$ & $\alpha_{8}$ & $a$ \\
\hline 1.0780 & 1.1629 & 0.9646 & 1.0212 & 1.0292 & 0.9346 & 1.0234 & 1.1336 & 6 \\
\hline
\end{tabular}

Consequently, the flow stresses corresponding to this plastic work are employed for the characterization of Yld2000-2D model together with the measured $r$-value. All the input data and results are reported in Table 2 .

\subsection{VFM identification}

The VFM identification was conducted using double notched specimens cut from the same BH340 sheet metal used for the standard calibration, with the same geometry illustrated in Figure 4. As for the numerical study, three orientations were adopted, namely $0^{\circ}, 45^{\circ}$ and $90^{\circ}$ with respect to the RD. Both DIC measurement hardware and software are the same employed in Section 5.1 and all metrology settings adopted for the DIC are reported in Table 10

Figure 10 illustrates an example of the strain fields obtained from the experiments. The ROI was restricted to this zone between the two notches mainly for two reasons. First, this area remains planar up to large strains 


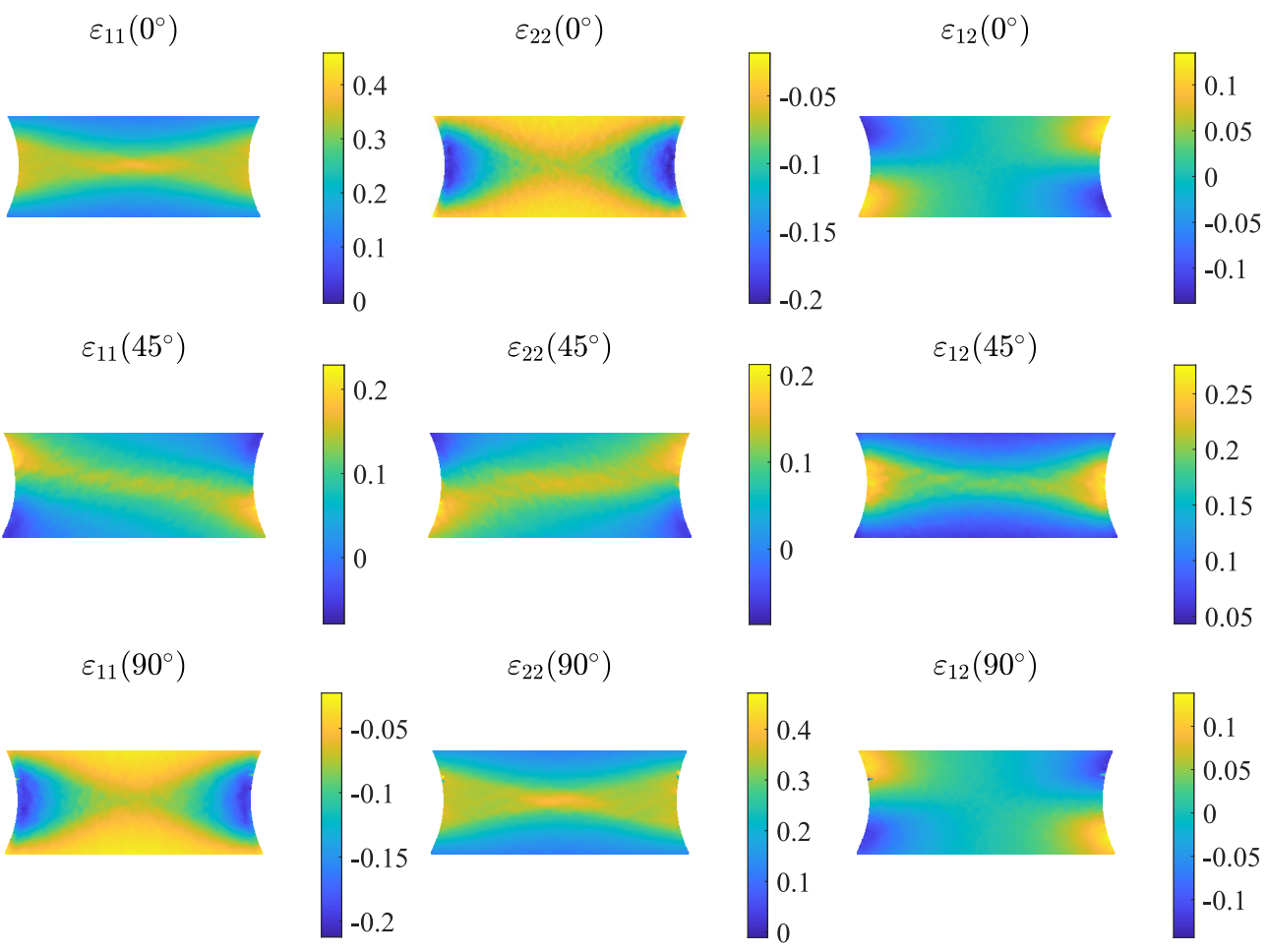

Figure 10: Logarithmic strain components in the material coordinate system on notched specimens at different material orientations obtained with DIC, at the last step considered for the VFM identification. 


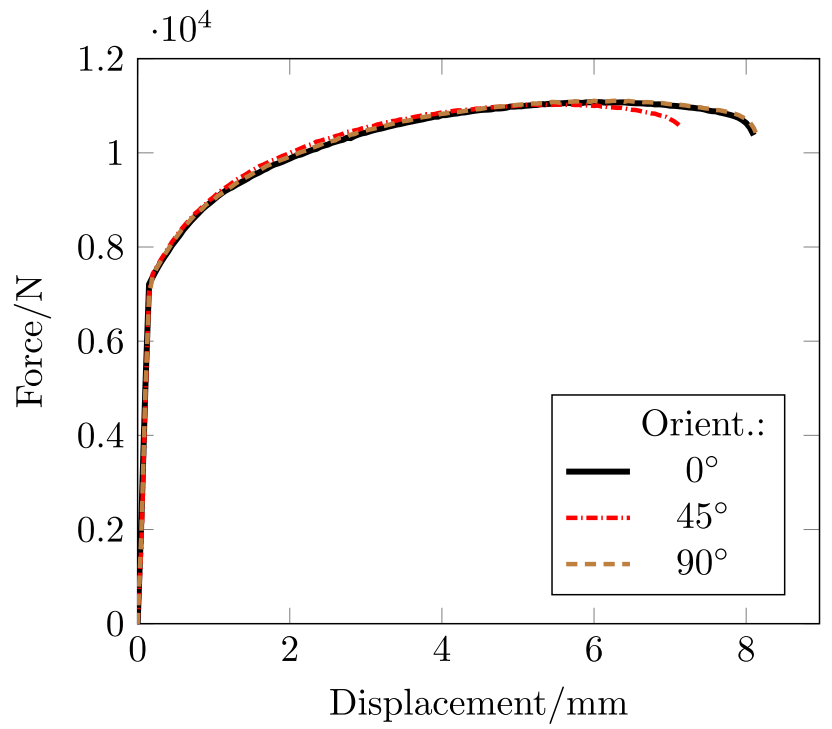

Figure 11: Measured loading force and displacement for the three notched specimens.

whereas the other areas of the specimen tend to wrinkle when load increases [31; second, since the plastic flow localizes within this zone reaching the highest deformations, the strain increment for each step is less affected by noise, which generally has the magnitude of the elastic strains [15]. The selected ROI consists of $146 \times 408$ measurement points.

Figure 11 shows the force vs displacement curves measured in the three notched tests. In this case, the three curves are almost similar, the increase in terms of force observed in the uniaxial test at $45^{\circ}$ is not significant in the corresponding notched specimen. Each test was subdivided in 22 load steps that were used in the inverse identification procedure. The results are presented and discussed in the following section. 


\section{Discussion}

The identification was conducted on three tests, using 22 load steps and $146 \times 408$ measurement points, loading to the processing of more than 1.3 million points were processed. In order to analyze the identification results, a study was conducted to evaluate the distribution of the measurement points in stress space.

\subsection{Analysis of the stress heterogeneity}

Figure $12 \mathrm{a}$ displays the stress state corresponding to each experimental measurement point as a dot in the normalized yield surface. The stress was computed from the strain field using the constitutive model and the reference parameters of Table 2. Using three notched specimens at three different material orientations allows to cover a diffused zone of the normalized $\pi$-plane first quadrant. However, the chart of Figure 12a does not provide a complete insight into the actual distribution of the heterogeneous stress fields, because not all zones are covered with the same intensity. To overcome this issue, a stress probability density function was defined dividing the normalized stress plane in uniform square bins and counting the number of points within each bin.

Figure $12 \mathrm{~b}$ depicts such function computed for the experimental stress points, showing that only some restricted portions of the yield surface present an high frequency of occurrences. Material points from specimens oriented at $0^{\circ}$ and $90^{\circ}$, are densely distributed closer to the plane strain region. Data points from the $45^{\circ}$ oriented specimen are densely distributed in a small area in the centre of the yield surface, which corresponds to the plane strain region 


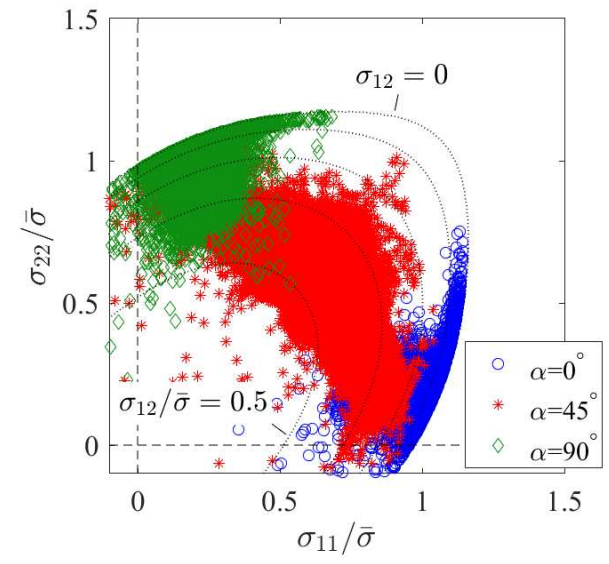

(a)

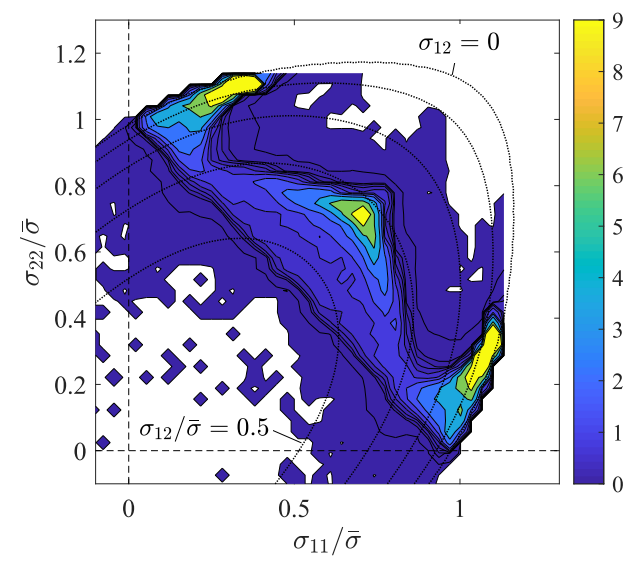

(b)

Figure 12: Distribution of the experimental stress states and Yld2000-2D yield surface (a) and corresponding probability density function contour plot (b).

oriented at $45^{\circ}$.

The same approach was used to study the stress distribution at different equivalent plastic strain increments in order to observe the stress field evolution on the yield surface during the loading path. Considering an equivalent plastic strain range $0 \leq \bar{\varepsilon}_{p} \leq 0.15$ (Figure 13a), the stress data points are scattered accordingly to the trend in Figure $12 \mathrm{~b}$; when the $\bar{\varepsilon}_{p}$ range is increased up to 0.3 (Figure 13b), the corresponding stress states tends to spread out closer to the respective specimens' material orientation. Finally, the probability density of stress points at $\bar{\varepsilon}_{p}>0.3$ (Figure 13c) covers a smaller area compared to the previous cases. This analysis demonstrates that the stress heterogeneity tends to reduce as the plastic strain localize at the end of the test. 


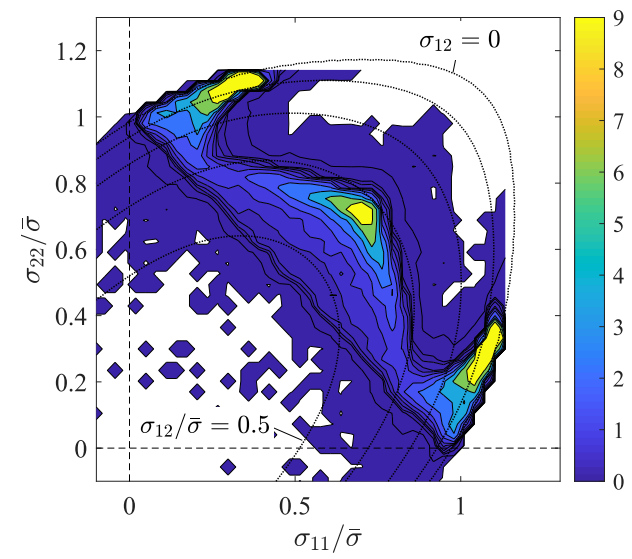

(a) $\bar{\varepsilon}_{p} \leq 0.15$

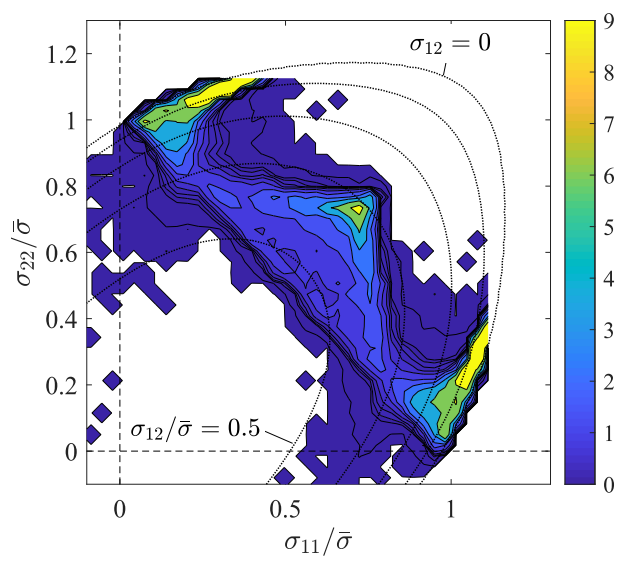

(b) $0.15<\bar{\varepsilon}_{p} \leq 0.3$

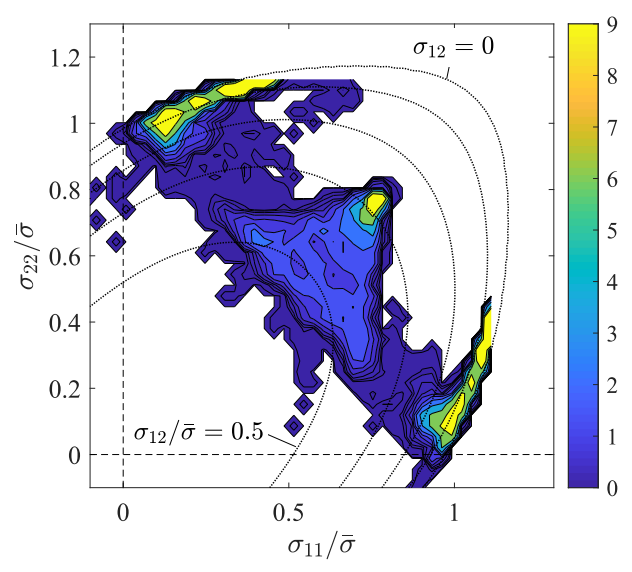

(c) $\bar{\varepsilon}_{p}>0.3$

Figure 13: Probability density function of the experimental stress states on the yield surface locus at different levels of accumulated equivalent plastic strain $\bar{\varepsilon}_{p}$. 


\subsection{Identification results}

In this section the identification results are discussed, first the identification of the hardening curve by LSSCI is presented, then the results of the non-linear VFM are discussed considered different combination of VFs and experimental tests.

\subsubsection{Identification of the hardening curve and smoothing of the data}

According to the adopted identification strategy, the LSSCI is the first step that provides the material hardening behaviour by means of a linear piecewise function. The hardening was identified from a single test, i.e. the notched specimen cut at $0^{\circ}$ from the RD. Furthermore, this step was also exploited to define the level of smoothing to apply on the measured strain data.

Experimental uncertainties of the DIC measurement play a crucial role in the VFM identification. Noisy strain data can represent, in fact, a considerable issue, as reported in [51 -53] for elasticity or in [29, 31] for anisotropic plasticity. For this reason, both temporal and spatial smoothing were applied on the experimental data, using the least-square smoothing method based on the convolution algorithm proposed by Gorry [54]. The temporal smoothing was performed using 7 time steps. The size of the strain window used for the spatial smoothing, instead, was chosen using the LSSCI method as diagnostic tool. Figure 14 reports the hardening curve identified using different levels of spatial smoothing. The hardening curve stabilizes using a strain windows of $9 \times 9$ points, i.e. increasing the level of smoothing does not produce variations in the identified curve. Therefore, a strain window of $9 \times 9$ points was used to process the experimental data input in the following VFM identification. 


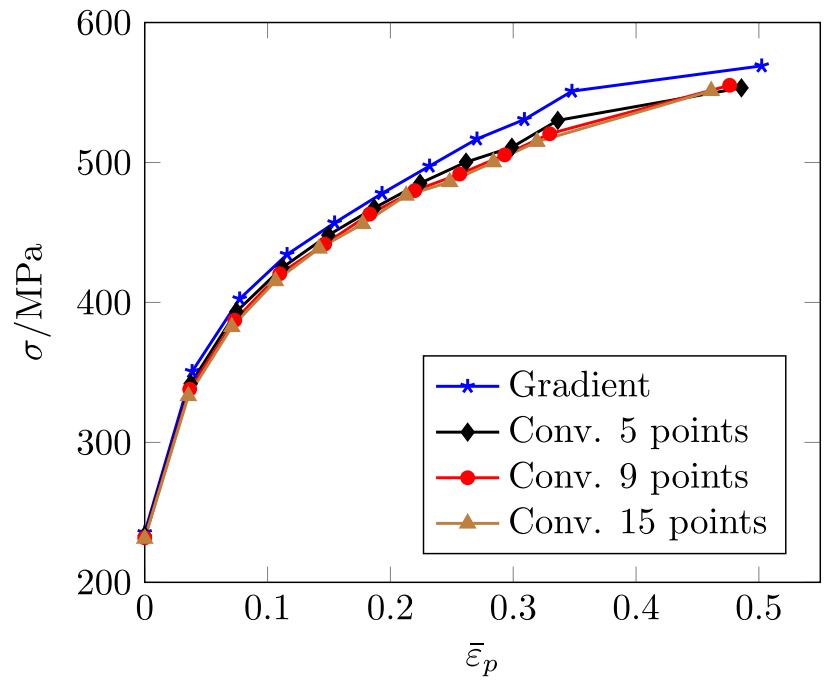

Figure 14: Identified piecewise linear hardening curve with LSSCI method using experimental data from notched specimen at $0^{\circ}$.

\subsubsection{Generation of Sensitivity Based Virtual Fields}

Although the SBVFs are automatically defined through the stress sensitivity, as already discussed in Section 2.3, their generation requires the definition of few parameters which can interact with the experimental uncertainties. So, in order to define their optimal configuration, each parameter governing the SBVFs generation was varied for a competitive evaluation of the identification results. The investigated aspects are the size of the virtual mesh, the effect of the perturbation parameter $\delta \xi_{j}$ and difference between the total (TSS, Eq. 16) and the incremental (ISS, Eq. 17) formulation of the SBVFs.

Figure 15 illustrates the identification results obtained using 3 sizes of the mesh grid and 2 values of the perturbation parameter. The incremental formulation was always used. A good agreement with the standard calibration 

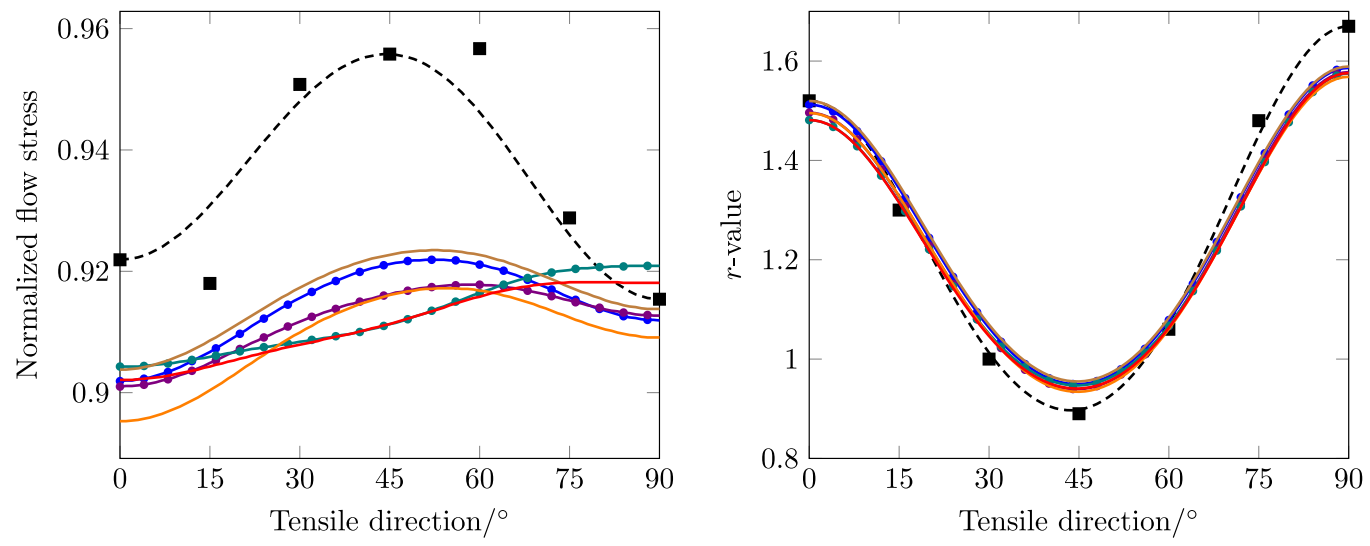

- Experimental $\multimap \delta \xi_{j}=0.15 ; 5 \times 5 \multimap \delta \xi_{j}=0.15 ; 20 \times 20-\delta \xi_{j}=0.20 ; 15 \times 15$

---- Standard cal. $\rightarrow \delta \xi_{j}=0.15 ; 15 \times 15-\delta \xi_{j}=0.20 ; 5 \times 5-\delta \xi_{j}=0.20 ; 20 \times 20$

Figure 15: Effect of perturbation parameter $\delta \xi_{j}$ on the prediction of anisotropy of uniaxial yield stress and $r$-value using different SBVFs virtual mesh size.

was always obtained in terms of $r$-values, instead a difference is observed in the identification of the normalized flow stress. In particular, the increase of the normalized flow stress at $45^{\circ}$ is not observed using the $20 \times 20$ mesh grid while better predictions are obtained using the coarser mesh grids. This improvement can be linked to the mitigation of the experimental noise due to the less dense distribution of nodes. The better identification was obtained using the $5 \times 5$ mesh with a maximum difference between the experimental points and the predicted ones of around 5\%. The variation of the perturbation parameter $\delta \xi_{j}$ does not produce significant changes in the identified results.

Figure 16 illustrates the difference in the identification achieved using the total (TSS) or the incremental (ISS) SBVFs, different sizes of the virtual mesh are also taken into consideration. As previously, the $r$-values are always identified correctly, however, the TSS outperforms the ISS, allowing a better 

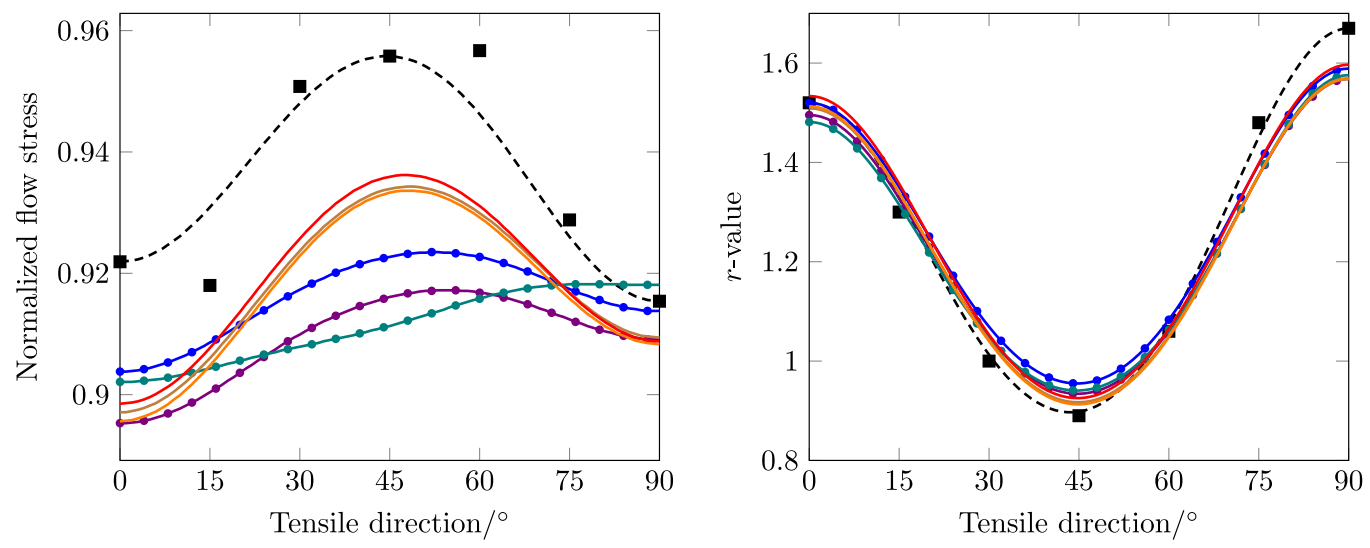

- Experimental $\multimap$ ISS; $5 \times 5 \longrightarrow$ ISS; $20 \times 20-$ TSS; $15 \times 15$

---- Standard cal. $\rightarrow$ ISS; $15 \times 15-$ TSS; $5 \times 5 \quad$ - TSS; $20 \times 20$

Figure 16: Comparison of predicted uniaxial yield stress and $r$-value adopting total stress sensitivity maps (TSS) and incremental stress sensitivity maps (ISS) to built the SBVFs, at different virtual mesh size.

reproduction of the stress anisotropy and a reduction of the error at $45^{\circ}$ below $3 \%$. Moreover, Figure 16 indicates that the effect of mesh size previously observed is not valid when the TSS is used to generate the SBVFs. In this case, the results are only slightly affected by the mesh size, with the better outcome achieved by the $20 \times 20$ mesh. Probably, the TSS formulation provides strain sensitivity maps where the noise is intrinsically mitigated, canceling the homogenization effect given by a coarser grid. This abet to increase the resolution of the VFs by using a more dense virtual mesh, and, consequentially, to capture fine features of the stress sensitivity.

\subsubsection{Comparison with standard non-linear VFM identification framework}

As a final analysis, the performances of the decoupled identification scheme was compared with the common non-linear VFM framework, where the inverse characterization involves simultaneously the identification of the consti- 
tutive parameters for hardening and anisotropy. The Swift law was employed to describe the hardening curve, thereby, the minimization process involves now 11 variables. The initial estimate was chosen arbitrarily (see Table 7 in Appendix). In addition, the two approaches were evaluated employing both the UDVFs and the SBVFs, the latter with the settings that gave the best identification in the previous section (i.e. TSS, $\Delta \xi_{j}=0.20,20 \times 20$ grid).

As illustrated in Figure 17, when SBVFs are used in the non-linear VFM, the full approach and the decoupled strategy proposed in this paper give the same outcome. On the other hand, when UDVFs are used, the full identification method produces a worse prediction, while the decoupled method gives reasonably accurate results. The last observation suggest that the decoupled procedure is more robust that the standard procedure where hardening and anisotropy are combined in the same cost function.

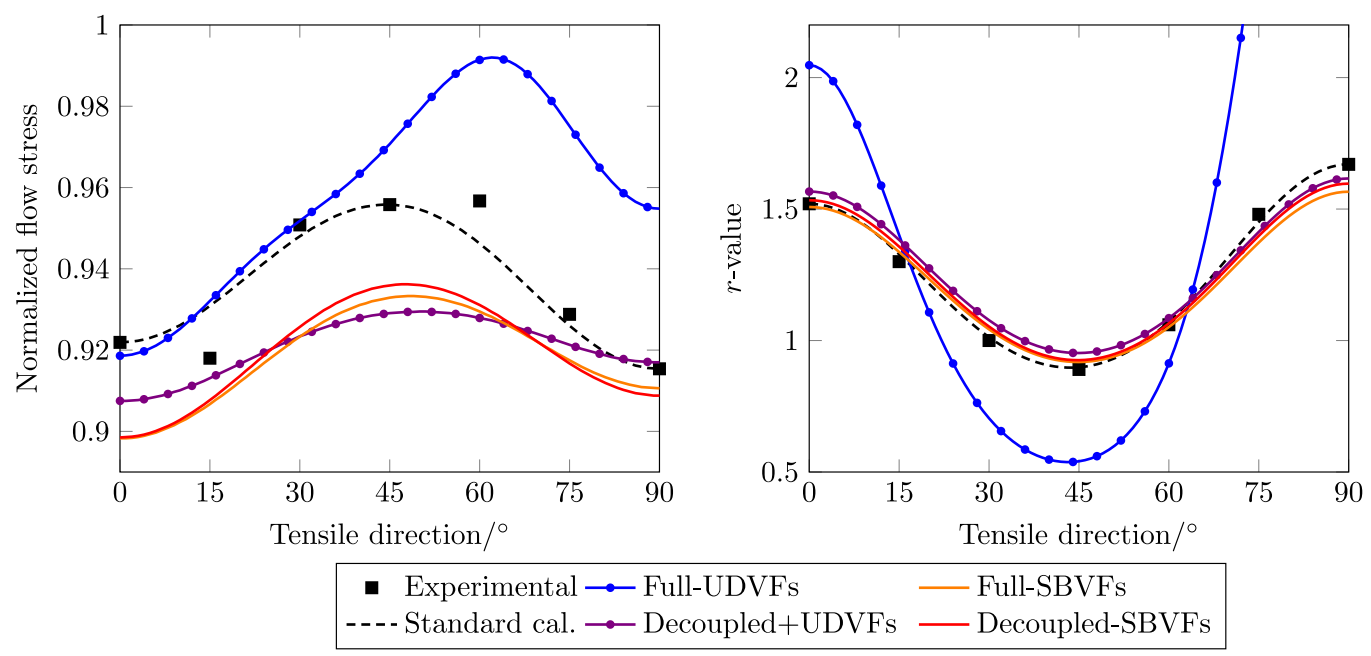

Figure 17: Predicted anisotropy of flow stress and $r$-value using the standard non-linear VFM identification scheme and the proposed decoupled strategy with different VFs types.

It is worth noting that, according to the numerical validation in Section 4 , 
there is not a relevant difference between UDVFs and SBVFs when tests at three different material orientation are adopted. Instead, in case of actual experimental data, the SBVFs seems to perform better than UDVFs, probably because of they are better suited to handle experimental uncertainties that were not introduced in the numerical validation.

Eventually, Figure 18 reports the normalized yield surfaces resulting from the standard calibration and the inverse identification obtained with the proposed decoupled method and the SBVFs, there is a remarkable agreement between the two surfaces. Moreover, albeit there are no direct stress data points from the balanced-biaxial state included in the VFM cost function (Figure 12a), feeding the identification with heterogeneous experimental strain fields from notched tensile specimens at three material orientations is sufficient to give an adequate prediction of the biaxial stress state.

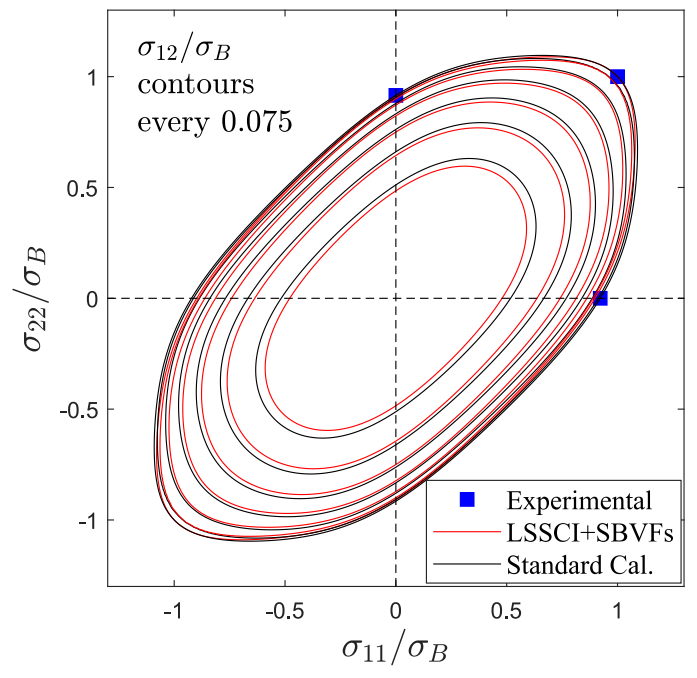

Figure 18: Yield surfaces by employing the standard procedure and the proposed decoupled identification strategy, normalized by their respective balanced biaxial stresses. 


\subsubsection{Identification from $45^{\circ}$ specimen data}

A further study was conducted trying to identify all parameters from a single test. In fact, according to the numerical validation of Section 4 , the application of SBVFs allows to identify the Yld2000-2D parameters feeding the VFM only with data from a single notched specimen at $45^{\circ}$, with a maximum error on the $r$-value below the $10 \%$. When the same approach is applied to experimental data, unfortunately neither UDVFs nor SBVFs permits to identify the correct anisotropic behaviour of BH340 (Figure 19). In this case, noise and other experimental uncertainties deteriorate the material information making the identification process necessitous of a larger set of data.
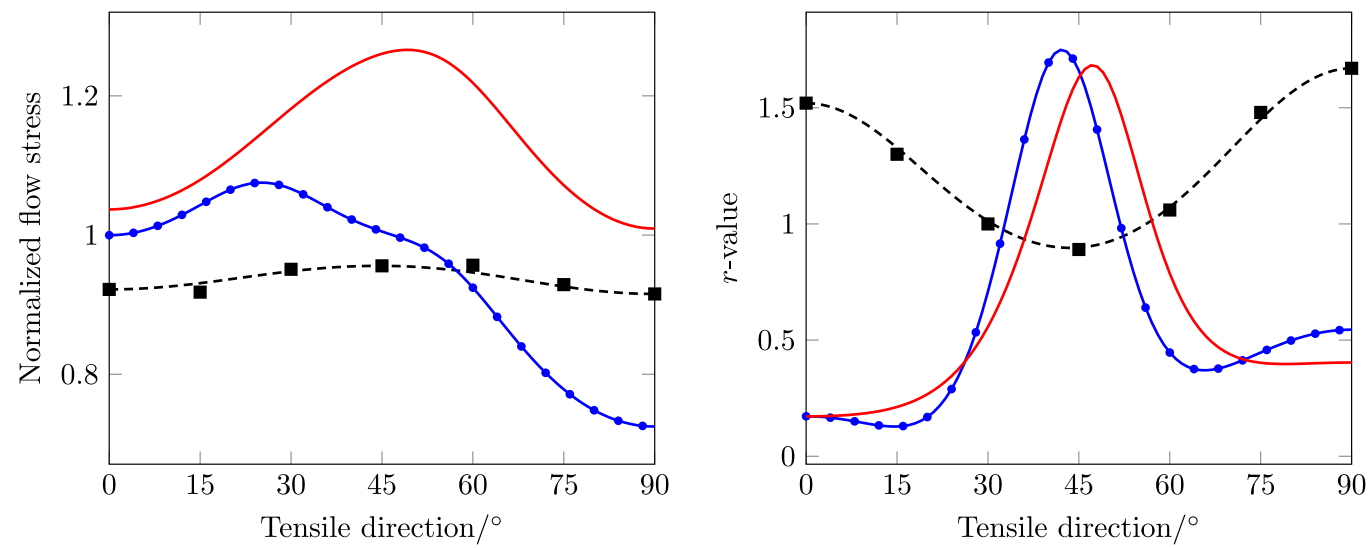

- Experimental $\longrightarrow$ UDVFs -- Standard cal. _ SBVFs

Figure 19: Comparison between UDVFs and SBVFs results employing only strain data at $45^{\circ}$ to predict the uniaxial yield stress and $r$-value anisotropy. 


\section{Computational time}

A reduced computational time represents an important aspect for industrial applications of inverse methods. All analysis reported in this paper were performed using a standard workstation equipped with an Intel $\AA$ Xeon $\AA$ CPU E5-1650 v2 @ $3.50 \mathrm{GHz}$ and $128 \mathrm{~GB}$ of RAM, while all routines were written in Matlabß language. The computational efficiency was assessed considering each processes of the flowchart in Figure 3 individually:

- the identification of the material hardening behaviour took an average of $1.5 \mathrm{~s}$ because of the linear piecewise formulation of LSSCI method, that become the most efficient step of the decoupled strategy chain;

- the SBVFs generation represents a computationally expensive operation due to the calculation of stress sensitivity and the reconstruction of piecewise virtual fields. For example, using the experimental strain data used in this paper, the creation of a SBVFs related to a single constitutive parameter was accomplished in almost $53 \mathrm{~s}$, completing the construction of the whole set of SBVFs for YLD2000-2D model in almost $7 \mathrm{~min}$;

- inverse calibration of the anisotropic plasticity model usually represents the most time consuming task, due to the iterative minimization process of the non-linear VFM cost function. Therefore, in order to speed up the calculation taking advantage of the multi-core architecture of the processor, the LMA optimization was parallelized on 6 cores. Table 3 summarizes the CPU time required to perform the identification 
of YLD2000-2D model on the BH340 steel, by involving different combinations of VFs and identification strategies. Decoupling the hardening behaviour enhances the computational efficiency for both SBVFs and UDVFs, by reducing the number of iterations to reach the solution. Moreover, experimental data only at $45^{\circ}$ specimen induces the minimization algorithm to increase the amount of iterations to find the local minimum, due to the lack of material information to constrain the minimization problem.

Table 3: Computational time evaluation results from non-linear VFM minimization.

\begin{tabular}{lcc}
\hline & Time [min] & Iterations \\
\hline Full-UDVFs, 3 tests & 19 & 126 \\
Full-SBVFs, 3 tests & 22 & 136 \\
Decoupled-UDVFs, 3 tests & 11 & 64 \\
Decoupled-SBVFs, 3 tests & 16 & 82 \\
Decoupled-SBVFs, 45 & 82 & 493 \\
\hline
\end{tabular}

\section{Conclusions}

In this paper, a VFM-based identification strategy is proposed for the inverse calibration of anisotropic plasticity models, investigating its capabilities to efficiently identify the constitutive parameters of an advanced anisotropic yield function: Yld2000-2D. Basically, the idea is to separate the identifications of the hardening behaviour and anisotropic yield function, where the 
former is achieved by the Linear Stress-Strain Curve Identification method (LSSCI), and the latter is assigned to the non-linear VFM.

The performance of the adopted scheme was evaluated initially on simulated tensile tests of deep notched specimens with longitudinal axes in three material orientations, and replicated in real experiments on BH340 steel. Moreover, the identification results were compared with the standard characterization procedure, which must include information from the biaxial stress state.

The study also focuses on the dependency of the results from the type of virtual fields employed in the VFM minimization, showing that the sensitivity based virtual fields lead to an accurate prediction of the material anisotropy.

This investigation leads to the following main conclusions:

- compared to standard applications of non-linear VFM, the separate identification of hardening behaviour of the yield function effectively enhance the results and reduces the computational time to achieve the solution. In fact, in the optimization problem given by the non-linear VFM, under equal constraints conditions, the decoupled scheme allows the elimination of the extra "degrees of freedom" linked to the variables regulating the hardening behaviour. This leads the minimization algorithm to focus on a reduced set of constitutive coefficients, requiring less iterations for convergence.

- Dealing with experimental uncertainties represents a relevant issue for inverse identifications, especially due to the intrinsic and chaotic nature of noise. Practically, there are not definite protocols that can help to suitably preprocess the strain data yet. In this paper, the LSSCI 
method was employed as a diagnostic tool to rapidly evaluate the quality of experimental data, and, thus, to apply the adequate smoothing.

- The sensitivity-based virtual fields (SBVFs) can considerably improve the results with respect to the user-defined virtual fields (UDVFs). In particular, the SBVFs looks to behave better in the case of experimental data. Moreover, their generation settings (i.e. virtual mesh size, $\Delta \xi_{j}$ and formulation) affect the identification, and must be opportunely defined to improve the results. According to the presented results, the total formulation seems to work better than the incremental one.

- The proposed identification method was successfully applied to the inverse calibration of the Yld2000-2D yield function, using heterogeneous strain data from double notched tensile specimens machined at three material orientations. The identified coefficients lead to a reasonable reproduction of the anisotropy of the biaxial stress state, thus, reducing the experimental effort to calibrate such complex material model. Nevertheless, such conclusion has been proved only for the BH340 steel and is not general. In this sense, the definition of the minimum amount of material information required to identify a material model represents a key issue for inverse methods, and may depends on the material itself. Therefore, further investigations should be made employing more anisotropic materials.

- To verify the heterogeneity of the experimental stress field, a probability density function of the stress distribution in the stress plane was introduced. This concept can be exploited in future works to design 
heterogeneous tests more effective than the double notched specimen.

\section{Acknowledgements}

The authors are grateful to $\mathrm{POSCO}{ }^{\circledR}$ for providing the material employed in the experiments.

\section{Appendix A}

In the following, the numerical values of the Yld2000-2D coefficients identified in this paper are listed. In particular, Table 4 contains the coefficients obtained in the numerical validation (Section 4); Table 5 and Table 6lists the coefficients obtained in the experiments using the SBVFs and the UDVFs, respectively; Table 7 lists the outcomes obtained with the full non-linear VFM.

Table 4: Yld2000-2D coefficients by using the decoupled identification scheme with different VFs and numerical data sets.

\begin{tabular}{llcccccccc}
\hline Data set: & VFs: & $\alpha_{1}$ & $\alpha_{2}$ & $\alpha_{3}$ & $\alpha_{4}$ & $\alpha_{5}$ & $\alpha_{6}$ & $\alpha_{7}$ & $\alpha_{8}$ \\
\hline \multirow{3}{*}{3 tests } & UDVFs & 1.0180 & 1.2271 & 1.0587 & 1.0124 & 0.9796 & 0.8738 & 1.0997 & 1.0780 \\
& SBVFs & 1.0237 & 1.2351 & 1.1232 & 1.0237 & 0.9884 & 0.9076 & 1.1098 & 1.0472 \\
\hline \multirow{2}{*}{ only 45 } & UDVFs & 0.7852 & 1.0503 & 0.756 & 0.9556 & 1.0148 & 1.0471 & 1.1117 & 0.9937 \\
& SBVFs & 0.9239 & 1.2377 & 1.2404 & 1.0052 & 0.9320 & 0.7241 & 1.0744 & 0.9908 \\
\hline
\end{tabular}




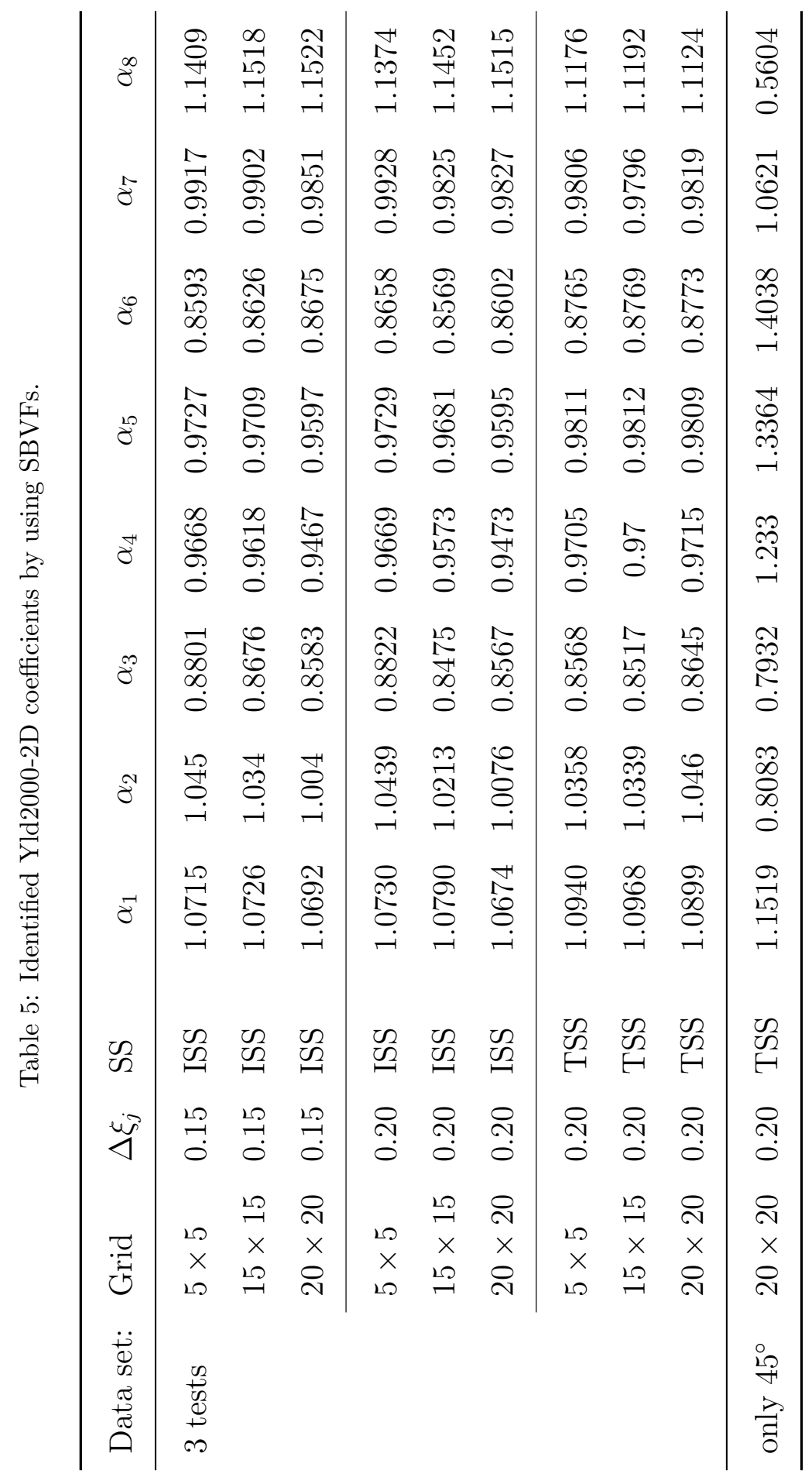



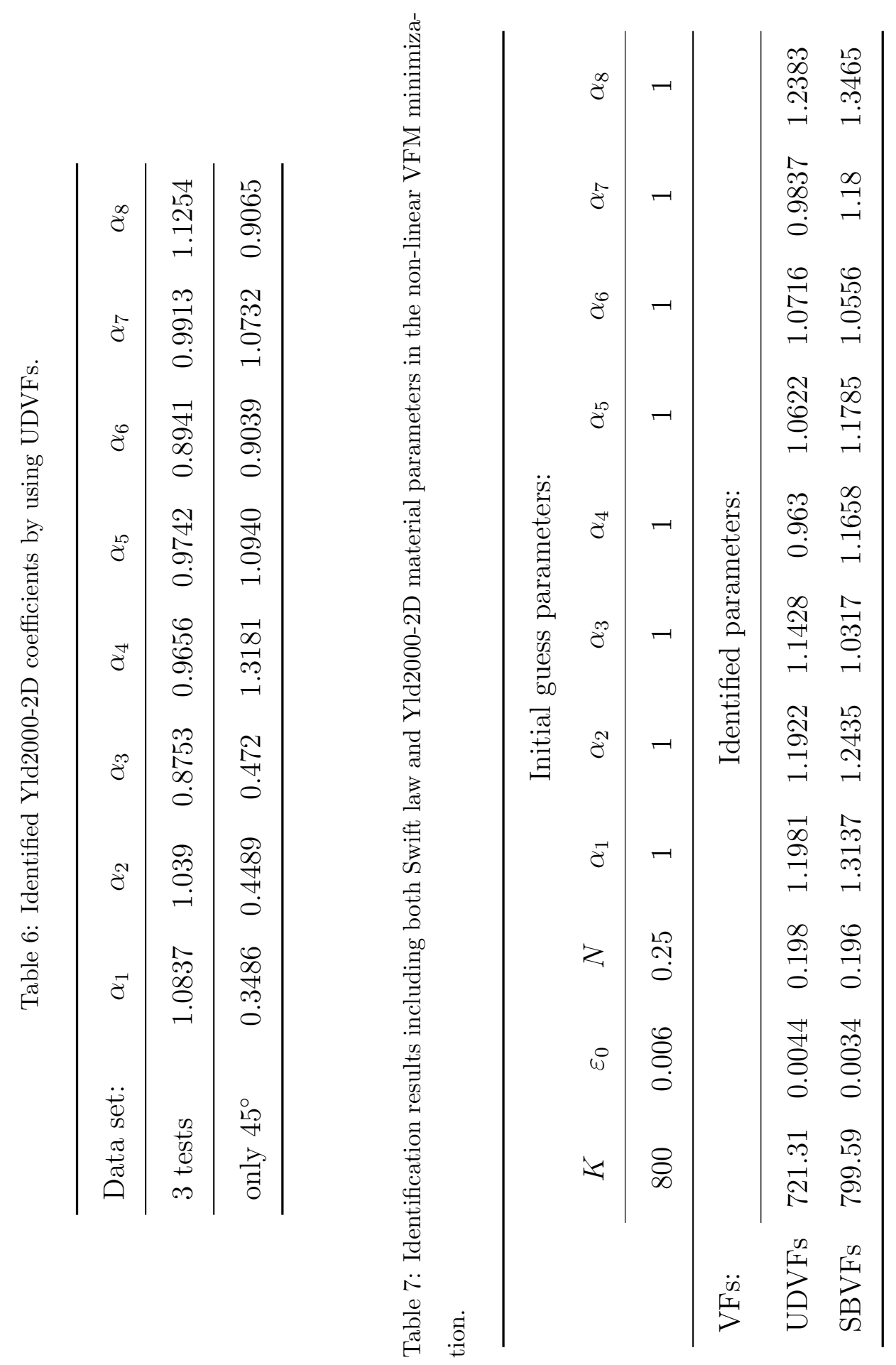
Appendix B 
Table 8: Adopted DIC settings for tensile tests on dogbone specimens.

\begin{tabular}{ll}
\hline Camera & Point Grey Grasshopper GRAS-50S5M-C \\
Image resolution & $2448 \times 2048,14$-bit \\
Field of view & $97.1 \times 81.3 \mathrm{~mm}$ \\
Pixel to mm conversion & 1 pixel=0.03968 mm \\
Stereo-angle & $21^{\circ}$ \\
Stand-off distance & $153 \mathrm{~mm}$ \\
Patterning Technique & Matt white spray paint base coat with black speckles \\
Pattern feature size (approx.) & 3.3 pixel / 0.13 mm \\
\hline DIC technique & Stereo correlation \\
DIC software & MatchID, version 2018.2.2 \\
Image Filtering & Gaussian, $5 \times 5$ pixel kernel \\
Subset size & 21 pixels / $0.83 \mathrm{~mm}$ \\
Step size & 3 pixels / 0.12 mm \\
Subset shape function & Affine \\
Matching criterion & Zero-normalized sum of square differences (ZNSSD) \\
Interpolant & Bi-cubic spline \\
Stereo transformation & Affine \\
Strain computation & Gradient \\
Spatial smoothing & Local polynomial regression Q8, 13 $\times 13$ window \\
Temporal smoothing & N/A \\
Virtual Strain Gauge Size & 57 pixels / $2.26 \mathrm{~mm}$ \\
Displacement noise floor & 0.03 pixels / $1.2 \mu m$ (in plane); 0.16 $\mu m$ (out-of-plane) \\
Strain (Hencky) noise-floor & $1.26 \times 10^{-5}$ \\
\hline
\end{tabular}


Table 9: Adopted DIC settings for the bulge test.

\begin{tabular}{|c|c|}
\hline Camera & Point Grey Grasshopper GRAS-50S5M-C \\
\hline Image resolution & $2448 \times 2048,14$-bit \\
\hline Field of view & $315.8 \times 264.25 \mathrm{~mm}$ \\
\hline Pixel to mm conversion & 1 pixel $=0.12903 \mathrm{~mm}$ \\
\hline Stereo-angle & $12.4^{\circ}$ \\
\hline Stand-off distance & $650 \mathrm{~mm}$ \\
\hline Patterning Technique & Matt white spray paint base coat with black speckles \\
\hline Pattern feature size (approx.) & 4.2 pixel / $0.54 \mathrm{~mm}$ \\
\hline DIC technique & Stereo correlation \\
\hline DIC software & MatchID, version 2018.2.2 \\
\hline Image Filtering & Gaussian, $5 \times 5$ pixel kernel \\
\hline Subset size & 43 pixels / $5.29 \mathrm{~mm}$ \\
\hline Step size & 7 pixels / $0.90 \mathrm{~mm}$ \\
\hline Subset shape function & Affine \\
\hline Matching criterion & Zero-normalized sum of square differences (ZNSSD) \\
\hline Interpolant & Bi-cubic spline \\
\hline Stereo transformation & Affine \\
\hline Strain formulation & Hencky logarithmic \\
\hline Spatial smoothing & Local polynomial regression $\mathrm{Q} 8,15 \times 15$ window \\
\hline Temporal smoothing & $\mathrm{N} / \mathrm{A}$ \\
\hline Virtual Strain Gauge Size & 139 pixels / $17.93 \mathrm{~mm}$ \\
\hline Displacement noise floor & 0.008 pixels / $0.28 \mu m$ (in plane); $2.0 \mu m$ (out-of-plane) \\
\hline Strain (Hencky) noise-floor & $1.65 \times 10^{-5}$ \\
\hline
\end{tabular}


Table 10: Adopted DICsettings for tensile tests on deep-notched specimens. For more information on the strain calculation and the adopted smoothing parameters, the reader is referred to Section 6.2.1.

\begin{tabular}{|c|c|}
\hline Camera & Point Grey Grasshopper GRAS-50S5M-C \\
\hline Image resolution & $2448 \times 2048,14$-bit \\
\hline Field of view & $93.6 \times 78.3 \mathrm{~mm}$ \\
\hline Pixel to mm conversion & 1 pixel $=0.038226 \mathrm{~mm}$ \\
\hline Stereo-angle & $22.6^{\circ}$ \\
\hline Stand-off distance & $151 \mathrm{~mm}$ \\
\hline Patterning Technique & Matt white spray paint base coat with black speckles \\
\hline Pattern feature size (approx.) & 3.8 pixels / $0.14 \mathrm{~mm}$ \\
\hline DIC technique & Stereo correlation \\
\hline DIC software & MatchID, version 2018.2.2 \\
\hline Image Filtering & Gaussian, $5 \times 5$ pixels kernel \\
\hline Subset size & 25 pixels / $0.96 \mathrm{~mm}$ \\
\hline Step size & 3 pixels / $0.11 \mathrm{~mm}$ \\
\hline Subset shape function & Irregular \\
\hline Matching criterion & Zero-normalized sum of square differences (ZNSSD) \\
\hline Interpolant & Bi-cubic spline \\
\hline Stereo transformation & Affine \\
\hline Strain formulation & Hencky logarithmic \\
\hline Strain computation & Least-squares differentiation by the Convolution Method \\
\hline Spatial smoothing & Savitzky-Golay, $9 \times 9$ window \\
\hline Temporal smoothing & Savitzky-Golay, 7 time steps window \\
\hline Virtual Strain Gauge Size & 49 pixels / $1.87 \mathrm{~mm}$ \\
\hline Displacement noise floor (not smoothed) & 0.006 pixels / $0.24 \mu \mathrm{m}$ (in plane); $0.34 \mu \mathrm{m}$ (out-of-plane) \\
\hline Strain noise-floor (not smoothed) & $8.43 \times 10^{-4}$ \\
\hline
\end{tabular}




\section{References}

\section{References}

[1] P.D. Wu, S.R. MacEwen, D.J. Lloyd, P. Tugcu, and K.W. Neale. On pre-straining and the evolution of material anisotropy in sheet metals. International Journal of Plasticity, 21:723-739, 2005.

[2] Jacek J. Skrzypek and Artur W. Ganczarski. Mechanics of anisotropic materials. Springer, 2015.

[3] M. Życzkowksky. Handbook of Materials Behaviour Models, chapter Anisotropic yield conditions, pages 155-164. Academic Press, 2001.

[4] F. Barlat, D. J. Lege, and J. C. Brem. A six-component yield function for anisotropic materials. International Journal of Plasticity, 7:693-712, 1991.

[5] F. Barlat, R. C. Becker, Y. Hayashida, Y. Maeda, M. Yanagawa, K. Chung, J. C. Brem, D. J. Lege, K. Matsui, S. J. Murtha, and S. Hattori. Yielding description for solution strengthened aluminium alloys. International Journal of Plasticity, 13(4):385-401, 1997.

[6] F. Barlat, Brem J.C., J.W. Yoon, K. Chung, R.E. Dick, D.J. Lege, F. Pourboghrat, S.H. Choi, and E. Chu. Plane stress yield function for aluminum alloy sheets - part 1: theory. International Journal of Plasticity, 19:1297-1319, 2003.

[7] F. Barlat, H. Aretz, J.W. Yoon, M.E. Karabin, J.C. Brem, and R.E. 
Dick. Linear transfomation-based anisotropic yield functions. International Journal of Plasticity, 21:1009-1039, 2005.

[8] O. Cazacu, B. Plunkett, and F. Barlat. Orthotropic yield criterion for hexagonal closed packed metals. International Journal of Plasticity, 22:1171-1194, 2006.

[9] J.W. Yoon, F. Barlat, R.E. Dick, K. Chung, and Kang T.J. Plane stress yield function for aluminum alloy sheets - part II: FE formulation and its implementation. International Journal of Plasticity, 20:495-522, 2004.

[10] J.W. Yoon, F. Barlat, R.E. Dick, and M.E. Karabin. Prediction of six or eight ears in a drawn cup based on a new anisotropic yield function. International Journal of Plasticity, 22:174-193, 2006.

[11] J.-H. Yoon, O. Cazacu, J.W. Yoon, and R. E. Dick. Earing predictions for strongly textured aluminum sheets. International Journal of Mechanical Sciences, 52:1563-1578, 2010.

[12] D.C. Ahn, J.W. Yoon, and K.Y. Kim. Modeling of anisotropic plastic behaviour of ferritic stainless steel. International Journal of Mechanical Sciences, 51:718-725, 2009.

[13] N. Deng, T. Kuwabara, and Korkolis Y.P. Cruciform specimen design and verification for constitutive identification of anisotropic sheets. Experimental Mechanics, 55:1005-1022, 2015.

[14] K. Chen, M. Scales, S. Kyriakides, and E. Corona. Effects of anisotropy 
on material hardening and burst in the bulge test. International Journal of Solids and Structures, 82:70-84, 2016.

[15] M.A Sutton, J.J. Orteu, and Schreier H.W. Image correlation for shape, motion and deformation measurements. Springer, 2009.

[16] S. Avril, M. Bonnet, A.-S. Bretelle, M. Grédiac, F. Hild, F. Ienny, F. Latourte, D. Lemosse, P. Pagano, E. Pagnacco, and F. Pierron. Overview of identification methods of mechanical properties based on full-field measurements. Experimental Mechanics, 48(381-402), 2008.

[17] Michel Grédiac and François Hild. Full-field measurements and Identification in Solid Mechanics. Wiley, 2012.

[18] J.M.P Martins, A. Andrade-Campos, and S. Thuillier. Comparison of inverse identification strategies for constitutive mechanical models using full-field measurements. Iternational Journal of Mechanical Sciences, 145:330-345, 2018.

[19] J. Kajberg and G. Lindkwist. Characterisation of materials subjected to large strains by inverse modelling based on in-plane displacement fields. International Journal of Solids and Structures, 41:3439-3459, 2004.

[20] D. Lecompte, S. Cooreman, S. Coppieters, J. Vantomme, H. Sol, and D. Debruyne. Parameter identification for anisotropic plasticity model using digital image correlation. European Journal of Computational Mechanics, 18:393-418, 2009.

[21] K. Denys, S. Coppieters, M. Seefeldt, and D. Debruyne. Multi-DIC setup for the identification of a $3 \mathrm{~d}$ anisotropic yield surface of thick 
high strength steel using a double perforated specimen. Mechanics of Materials, 100:96-108, 2016.

[22] A. Güner, C. Soyarslan, A. Brosius, and A.E. Tekkaya. Characterization of anisotropy of sheet metals employing inhomogeneous strain fields for Yld2000-2d yield function. International Journal of Solids and Structures, 49:3517-3527, 2012.

[23] F. Mathieu, H. Leclerc, F. Hild, and S. Roux. Estimation of elastoplastic parameters via weighted FEMU and Integrated DIC. Experimental Mechanics, 2014.

[24] M. Bertin, F. Hild, and S. Roux. On the identifiability of Hill-1948 plasticity model with a single biaxial test on very thin sheet. Strain, 2017.

[25] S. Coppieters, S. Cooreman, H. Sol, P. Van Houtte, and D. Debruyne. Identification of the post-necking hardening behaviour of sheet metal by comparison of the internal and external work in the necking zone. Journal of Materials Processing Technology, 211(3):545-552, 2011.

[26] S. Coppieters and T. Kuwabara. Identification of post-necking hardening phenomena in ductile sheet metal. Experimental Mechanics, 54:1355-1371, 2014.

[27] F. Pierron and M. Grédiac. The Virtual Fields Method. Springer, 2012.

[28] M. Grédiac and F. Pierron. Applying the Virtual Fields Method to the identification of elasto-plastic constitutive parameters. International Journal of Plasticity, 22:602-627, 2006. 
[29] M. Rossi and F. Pierron. Identification of plastic constitutive parameters at large deformations from three dimensional displacement fields. Computational Mechanics, 49:53-71, 2012.

[30] J.H. Kim, F. Barlat, F. Pierron, and M.G. Lee. Determination of anisotropic plastic constitutive parameters using the Virtual Fields Method. Experimental Mechanics, 54:1189-1204, 2014.

[31] M. Rossi, F. Pierron, and M. Štamborská. Application of the Virtual Fields Method to large strain anisotropic plasticity. International Journal of Solids and Structures, 97-98:322-335, 2016.

[32] J.M.P Martins, A. Andrade-Campos, and S. Thuillier. Calibration of anisotropic plasticity models using a biaxial test and the virtual fields method. International Journal of Solids and Structures, 172-173:21-37, 2019 .

[33] F. Pierron, S. Avril, and V. The Tran. Extension of the Virtual Fields Method to elasto-plastic material identification with cyclic loads and kinematic hardening. International Journal of Solids and Structures, 47:2993-3010, 2010.

[34] J. Fu, F. Barlat, and J.-H. Kim. Parameter identification of the homogeneous anisotropic hardening model using the Virtual Fields Method. International Journal of Material Forming, 2015.

[35] S. Avril, M. Grédiac, and F. Pierron. Sensitivity of the Virtual Fields Method to noisy data. Computational Mechanics, 34(6):439-452, 2004. 
[36] A. Marek, F. M. Davis, M. Rossi, and F. Pierron. Extension of the sensitivity-based virtual fields to large deformation anisotropic plasticity. International Journal of Material Forming, 2018.

[37] M. Rossi, A. Lattanzi, and F. Barlat. A general linear method to evaluate the hardening behaviour of metals at large strain with full-field measurements. Strain, 54, 2018.

[38] Albrecht Bertram. Elasticity and Plasticity of Large Deformations. Springer, 2008.

[39] J.W. Yoon, D.Y. Yang, K. Chung, and F. Barlat. A general elasto-plastic finite element formulation based on incremental deformation theory for planar anisotropy and its application to sheet metal forming. International Journal of Plasticity, 15:35-67, 1999.

[40] J. H. Kim, A. Serpantié, F. Barlat, F. Pierron, and M.-G. Lee. Characterization of the post-necking strain hardening behavior using the Virtual Fields Method. International Journal of Solids and Structures, 50:3829-3842, 2013.

[41] A. Pérez-Foguet and F. Armero. On the formulation of closest-point projection algorithms in elastoplasticity - part II: Globally convergent schemes. International Journal for Numerical Methods in Engineering, 53:331-374, 2002.

[42] A Marek, F.M. Davis, and F. Pierron. Sensitivity-based virtual fields for the non-linear Virtual Fields Method. Computational Mechanics, 60:409-431, 2017. 
[43] R.W. Logan and W.F. Hosford. Upper-bound anisotropic yield locus calculations assuming $<111>$-pencil glide. International Journal of Mechanical Sciences, 22:419-430, 1980.

[44] Ł. Kowalewski and M. Gajewski. Assessment of Optimization Methods used to determine plasticity parameters based on DIC and Back Calculation Methods. Experimental Techniques, 43:385-396, 2019.

[45] N. Suoto, S. Thuillier, and A. Andrade-Campos. Design of an indication to characterize and classify mechanical tests for sheet metals. International Journal of Mechanical Sciences, 101-102:252-271, 2015.

[46] N. Suoto, A. Andrade-Campos, and S. Thuillier. Mechanical design of a heterogeneous test for material parameters identification. International Journal of Material Forming, 2016.

[47] F. Barlat, Y. Maeda, K. Chung, M. Yanagawa, J. C. Brem, Y. Hayashida, D. J. Lege, K. Matsui, S. J. Murtha, S. Hattori, R. C. Becker, and S. Makosey. Yield function development for aluminium alloy sheets. Journal of the Mechanics and Physics of Solids, 45(11/12):1727176, 1997.

[48] G. Gutsher, H.-C. Wu, G. Ngaile, and T. Altan. Determination of flow stress for sheet metal forming using the viscous pressure bulge (VPB) test. Journal of Materials Processing Technology, 146:1-7, 2004.

[49] J.Y. Lee, F. Barlat, R.H. Wagoner, and M.G. Lee. Balanced biaxial testing of advanced high strength steels in warm conditions. Experimental Mechanics, 53:1681-1692, 2013. 
[50] ISO 16808:2014 : Determination of biaxial stress-strain curve by means of bulge test with optical measuring systems, 2014 .

[51] M. Rossi and F. Pierron. On the use of simulated experiments in designing tests for material characterization from full-field measurements. International Journal of Solids and Structures, 49:420-435, 2012.

[52] M. Rossi, P. Lava, F. Pierron, D. Debruyne, and M. Sasso. Effect of DIC spatial resolution, noise and interpolation error on identification results with the VFM. Strain, 51:206-222, 2015.

[53] M. Badaloni, M. Rossi, G. Chiappini, P. Lava, and D. Debruyne. Impact of experimental uncertainties on the identification of mechanical material properties using DIC. Experimental Mechanics, 55(8):1411-1426, 2015 .

[54] A. Gorry. General least-squares smoothing and differentiation by the convolution (Savitzky-Golay) method. Anal. Chem., 62:570-573, 1990. 